\title{
Nonlinear 1-D stationary flows in multi-ion plasmas - sonic and critical loci - solitary and "oscillatory" waves
}

\author{
E. M. Dubinin ${ }^{1}$, K. Sauer ${ }^{1}$, and J. F. McKenzie ${ }^{1,2,3}$ \\ ${ }^{1}$ Max-Planck-Institute für Sonnensystemforschung, Lindau, Germany \\ ${ }^{2}$ School of Pure and Applied Physics, University of Natal, Durban, South Africa \\ ${ }^{3}$ University of California, Riverside, CA, USA
}

Received: 27 April 2006 - Accepted: 11 September 2006 - Published: 22 November 2006

\begin{abstract}
One-dimensional stationary flows of a plasma consisting of two ion populations and electrons streaming against a heavy ion cloud are studied. The flow structure is critically governed by the position of sonic and critical points, at which the flow is shocked or choked. The concept of sonic and critical points is suitably generalized to the case of multi-ion plasmas to include a differential ion streaming. For magnetic field free flows, the sonic and critical loci in the $\left(u_{p x}, u_{h x}\right)$ space coincide. Amongst the different flow patterns for the protons and heavy ions, there is a possible configuration composed of a "heavy ion shock" accompanied by a proton rarefaction. The magnetic field introduces a "stiffness" for the differential ion streaming transverse to the magnetic field. In general, both ion fluids respond similarly in the presence of "ion obstacle"; the superfast (subfast) flows are decelerated (accelerated). The collective flow is choked when the dynamic trajectory $\left(u_{p x}, u_{h x}\right)$ crosses the critical loci. In specific regimes the flow contains a sequence of solitary structures and as a result, the flow is strongly bunched. In each such substructure the protons are almost completely replaced by the heavies. A differential ion streaming is more accessible in the collective flows oblique to the magnetic field. Such a flexibility of the ion motion is determined by the properties of energy integrals and the Bernoulli energy functions of each ion species. The structure of flows, oblique to the magnetic field, depends critically on the velocity regime and demonstrates a rich variety of solitary and oscillatory nonlinear wave structures. The results of the paper are relevant to the plasma and field environments at comets and planets through the interaction with the solar wind.
\end{abstract}

Keywords. Magnetospheric physics (Plasma waves and instabilities; Solar wind interactions with unmagnetized bodies) - Space plasma physics (Nonlinear phenomena; Shock waves)

Correspondence to: E. M. Dubinin

(dubinin@mps.mpg.de)

\section{Introduction}

The multi-ion nature of the solar wind and planetary/cometary plasmas gives rise to new and interesting effects. The plasma and field environment of comets Halley and Grigg-Skjellerup encountered by the Giotto spacecraft displayed a complex structure characterized by the existence of unexpected sharp boundaries which separate regions with different plasma characteristics, which are not predicted by classical MHD models (see, e.g. Coates, 1997, and references therein). Space measurements near the nonmagnetized planets with extended ionospheric/atmospheric shells have also shown the appearance of unexpected boundaries (e.g. pile-up boundaries at Mars and Venus (Acuna et al., 1998; Bertucci et al., 2005; Boesswetter et al., 2004). Although the nature of these boundaries remains unclear, it is suggested that the multi-ion origin of the interacting plasmas may be a key element. Another interesting feature of the plasma environment of comets and nonmagnetized planets is the observations of strongly nonlinear coherent wave structures which often fill the broad regions of the interaction between different plasmas (Rème et al., 1987, 1993).

It is well known that the existence of discontinuities in plasmas is often related to transitions through "sonic" points. The appearance of new wave modes in a multiion plasma can modify the pattern of standing waves upstream of any effective obstacle and influence the position of "sonic" points. Moreover differential streaming between different ion species requires the concept of sonic point in a multi-fluid plasma to be generalized. McKenzie et al. (1992) and McKenzie (2001) have generalized the idea of a sonic point in differentially streaming, magnetic field-free, multiion plasmas by using the linear dispersion to find the condition that compressional waves, as viewed in a laboratory frame relative to which the various ion species stream, appear stationary.

In this study we consider the problem of sonic and critical points in the more complicated case of a magnetized multiion plasma. To clarify this issue we examine the structure of

Published by Copernicus GmbH on behalf of the European Geosciences Union. 
the one-dimensional stationary flows of plasma consisting of protons, heavy ions (e.g. $\mathrm{O}^{+}$) and electrons. The heavy (oxygen) ions form a local source which acts as an obstacle to the flow. In Sect. 2 we investigate the structure of the flow without magnetic field. It is shown that for certain parameters, a "heavy ion shock", corresponding to the "slow wave mode" accompanied by a rarefaction of the protons is generated in front of the obstacle. In Sect. 3 we include a magnetic field transverse to the plasma flow. In this case a sonic point translates to a locus in the $\left(u_{p x}, u_{h x}\right)$ plane, which corresponds to a superfast-subfast transition, and the value of speed which generalizes the fast magnetosonic speed in a bi-ion plasma, is evaluated. In contrast to flows without a magnetic field, the loci of critical points, on which $d u_{i} / d x=\infty$, do not coincide with the sonic locus determined from the condition of wave stationarity. The structure of the equation for the critical loci is similar to the magnetic field-free case and describes two loci in the $\left(u_{p x}, u_{h x}\right)$ plane. There are specific values of the Mach numbers at which solitary bi-ion structures are embedded in the flow. Finally, in Sect. 4 we examine the structure of flow oblique to the magnetic field. The problem of critical points is discussed in the terms of critical loci and also the characteristic features of the Bernoulli energy functions for bi-ion flows. It is shown that the characteristics of a flow may be strongly influenced by the possible existence of solitary and oscillatory structures.

\section{Magnetic field free flows}

\subsection{Governing equations}

We consider one-dimensional flow of a plasma consisting of protons $(p)$, heavier ion species $(h)$ and electrons $(e)$. The heavies are described by a local source which forms a "cloud" of ions, centered at $x=x_{o}$. The basic equations governing the ion dynamics are the standard multifluid equations of continuity and momentum:

$$
\begin{aligned}
& \frac{\partial n_{p}}{\partial t}+\frac{\partial}{\partial x}\left(n_{p} u_{p}\right)=0, \\
& \frac{\partial n_{h}}{\partial t}+\frac{\partial}{\partial x}\left(n_{h} u_{h}\right)=S(x), \\
& m_{i}\left(\frac{\partial u_{i}}{\partial t}+u_{i} \frac{\partial u_{i}}{\partial x}\right)=e Z_{i} E-\frac{\nabla p_{i}}{n_{i}} \quad(i=p, h) \\
& E=-\frac{1}{n_{e} e} \frac{\partial p_{e}}{\partial x}
\end{aligned}
$$

The latter equation prescribes massless electrons and under isothermal conditions yields the so called Bolzmann distribution for $n_{e}$.

$$
n_{e}=n_{o} e^{e \varphi / T_{e}}
$$

where $\varphi$ is the electric field potential $\left(\frac{\partial \varphi}{\partial x}=-E\right)$ and $T_{e}$ is the electron temperature in energy units.

We will investigate the steady-state response of the plasma flow on the "obstacle" for which purposes Eqs. (1), in dimensionless variables, for stationary flows $\left(\frac{\partial}{\partial t}=0\right)$ may be written as

$n_{p} u_{p}=1$

$n_{h} u_{h}=P \equiv \int_{-\infty}^{x} S(x) d x$

$\left(1-\frac{1}{M_{p}^{2} u_{p}^{2}}\right) \frac{d u_{p}}{d x}=-\frac{1}{M_{s}^{2}} \frac{1}{n_{e} u_{p}} \frac{d n_{e}}{d x}$,

$\left(1-\frac{1}{M_{h}^{2} u_{h}^{2}}\right) \frac{d u_{h}}{d x}=-\frac{1}{\mu M_{s}^{2}} \frac{1}{n_{e} u_{h}} \frac{d n_{e}}{d x}-\frac{1}{M_{h}^{2} u_{h} P} \frac{d P}{d x}$,

where $M_{s}, M_{p}, M_{h}$ are the Mach numbers characterizing the flow of different species, $M_{s}=\frac{u_{o}}{c_{s}}\left(c_{s}^{2}=T_{e} / m_{p}\right), M_{p}=\frac{u_{p o}}{c_{p}}$ $\left(c_{p}^{2}=T_{p} / m_{p}\right), M_{h}=\frac{u_{h o}}{c_{h}}\left(c_{h}^{2}=T_{h} / m_{h}\right), m_{p}=1, m_{h}=\mu$, the velocities are normalized to their values at $x=-\infty$ $\left(u_{o}=u_{p o}=u_{h o}=1, n_{p o}=1, n_{h o} u_{h o}=\alpha\right)$, i.e. at $x=-\infty$ both ion fluids stream from the left to right with equal speeds. For convenience, we also assume that the ions are isothermal. Note that the spatial variable $x$ is normalized to the value $x=x_{c}$ which characterizes the spatial extent of the source. Correspondingly, the electric field is normalized to the value $E_{o}=m_{p} u_{o}^{2} / e x_{c}$.

The spatial distribution of the source of heavy ions is taken as

$S=S_{o} e^{-\frac{\left|x-x_{o}\right|}{x_{c}}}$.

Then the heavy ion flux $P=n_{h} u_{h}$ is

$P=\left\{\begin{aligned} x_{c} S_{o} e^{\frac{\left(x-x_{o}\right)}{x_{c}}}+\alpha & : \quad x<x_{o} \\ x_{c} S_{o}\left(2-e^{-\frac{\left(x-x_{o}\right)}{x_{C}}}\right)+\alpha & : \quad x>x_{o}\end{aligned}\right.$

The relation between the number densities of the species is given by the quasineutrality condition

$n_{e}=\frac{1}{u_{p}}+\frac{P(x)}{u_{h}}$.

\subsection{Sonic and critical loci in a bi-ion plasma}

It is well known that the study of stationary flows is intimately related with the problem of sonic points. The problem of sonic loci in a multi-ion plasma was discussed in McKenzie et al. (1993), McKenzie (2001). Here we briefly recall some important results. In a single-ion plasma streaming 
with the speed $u_{o}$, the sonic point is met where the flow speed matches the sonic speed. Indeed Eqs. (1a) and (1c) with assumption that $n_{p}=n_{p}\left(u_{p}\right)$ yield

$\frac{\partial u_{p}}{\partial t}+\left(u_{p} \pm c_{s}\right) \frac{\partial u_{p}}{\partial x}=0$

With the sign minus, the compressional wave propagates to the left and the flow becomes stationary, $\frac{\partial}{\partial t}=0$ if the protons stream to the right with the sonic speed, which is the phase speed of waves in a single-ion plasma. In the rest frame the phase speed of a compressional wave propagating to the left is reduced to zero (the wave is carried to the right by the flow). Therefore the sonic point is reached when the compressional wave becomes stationary.

In general, in a multi-ion plasma, the speeds of ion fluids are different. To determine a "sonic" point in a such plasma configuration one has to derive the dispersion equations for waves propagating in a plasma with differentially streaming ions and then put $V_{p h} \equiv \omega / k=0$. Such a procedure gives the equation which generalizes the idea of the sonic point for multi-ion plasmas, namely

$$
\sum_{i} \frac{a_{i}^{2}}{u_{i}^{2}-c_{i}^{2}}=1
$$

where $u_{i}$ are the bulk speeds of the ion fluids, $c_{i}$ are the ion thermal speeds, $c_{i}^{2}=T_{i} / m_{i}$, and $a_{i}$ are the modified sound speeds, $a_{i}^{2}=\frac{n_{i}}{\mu_{i} n_{e}} c_{s}^{2}$. In a plasma, consisting of two ion species Eq. (8) is reduced to two sonic loci in the $u_{p}, u_{h}$ plane. The appearance of two loci is associated with two different compressional modes in a bi-ion plasma, fast and slow, given by

$V_{p h 1,2}^{2} \equiv \frac{\omega^{2}}{k^{2}}=\frac{c_{p}^{2}+c_{h}^{2}}{2} \pm \sqrt{\frac{\left(c_{p}^{2}-c_{h}^{2}\right)^{2}+4\left(a_{p}^{2}+a_{h}^{2}\right)}{4}}$

If the electrons are cold, $V_{p h 1,2}=c_{p}, c_{h}$. If the ions are cold, two modes merge to one with $V_{p h}=c_{s}\left(1-\frac{\mu-1}{\mu} \frac{n_{h}}{n_{e}}\right)^{1 / 2}$. In the general case, the "sonic point" is met where the combination of the ion speeds $\left(u_{p}, u_{h}\right)$ lies on two loci (Fig. 1a). The hyperbolic-like curve in the upper corner of the $u_{p}, u_{h}$ plane corresponds to the "superfast/subfast" transition related to the proton wave mode. The closed locus encircling the origin is associated with the slower heavy ion mode. Correspondingly, region 2 may be labeled "subfast", but "superslow", and region 3 "subslow".

If we take the plus sign in Eq. (7) (the sound wave propagates in the same direction as the plasma flow) and go to the reference frame moving to the right with the sound speed $c_{s}$, Eq. (7) reduces to a simple nonlinear equation for the ionsound wave $\frac{d u_{p}}{d t}=0$, where the velocities $u_{p}$ can be considered as perturbations to the wave speed $c_{s}$. In a such nonlinear sound wave all points move with a constant speed which

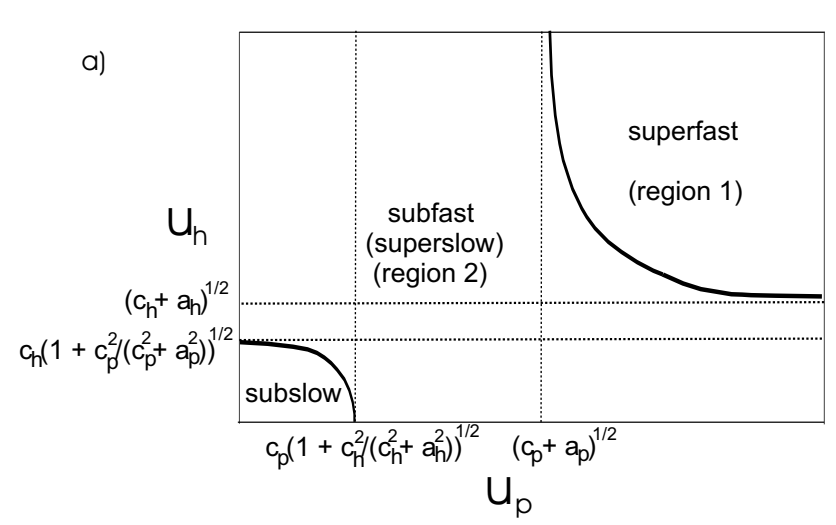

b)

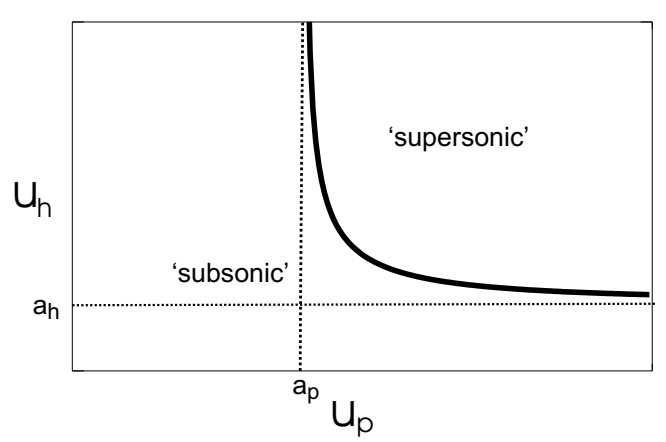

Fig. 1. (a) Sonic and critical loci in a bi-ion plasma without magnetic field, (b) two loci degenerate to one locus for the case of cold ions.

is proportional to the amplitude of the perturbation $u_{p}$. This admits a nonlinear wave steepening since points with higher $u_{p}$ move faster than points with smaller $u_{p}$. If we neglect dispersion and dissipation, steepening leads to $\frac{d u_{p}}{d x} \rightarrow \infty$ and overturning of the wave. If dissipation is included, a shock wave can be formed. Therefore, in a single-ion plasma sonic and critical points are reached where the flow becomes sonic.

In a bi-ion plasma, critical points (or loci) can be found from the condition

$\frac{d u_{i}}{d x} \rightarrow \infty$

Differentiating Eq. (6) and substituting into Eq. (3) yields two nonlinear differential equations in the form

$\frac{d u_{i}}{d x}=\frac{N_{i}}{D_{i}}$

where $N_{i}$ and $D_{i}$ are algebraic functions of $u_{i}, P(x)$ and the Mach numbers. We are interested in the denominators,

$D_{i}=\left(1-\frac{1}{M_{i}^{2} u_{i}^{2}}\right)\left(1-\frac{a_{p}^{2}}{\left(u_{p}^{2}-c_{p}^{2}\right)}-\frac{a_{h}^{2}}{\left(u_{h}^{2}-c_{h}^{2}\right)}\right)$ 

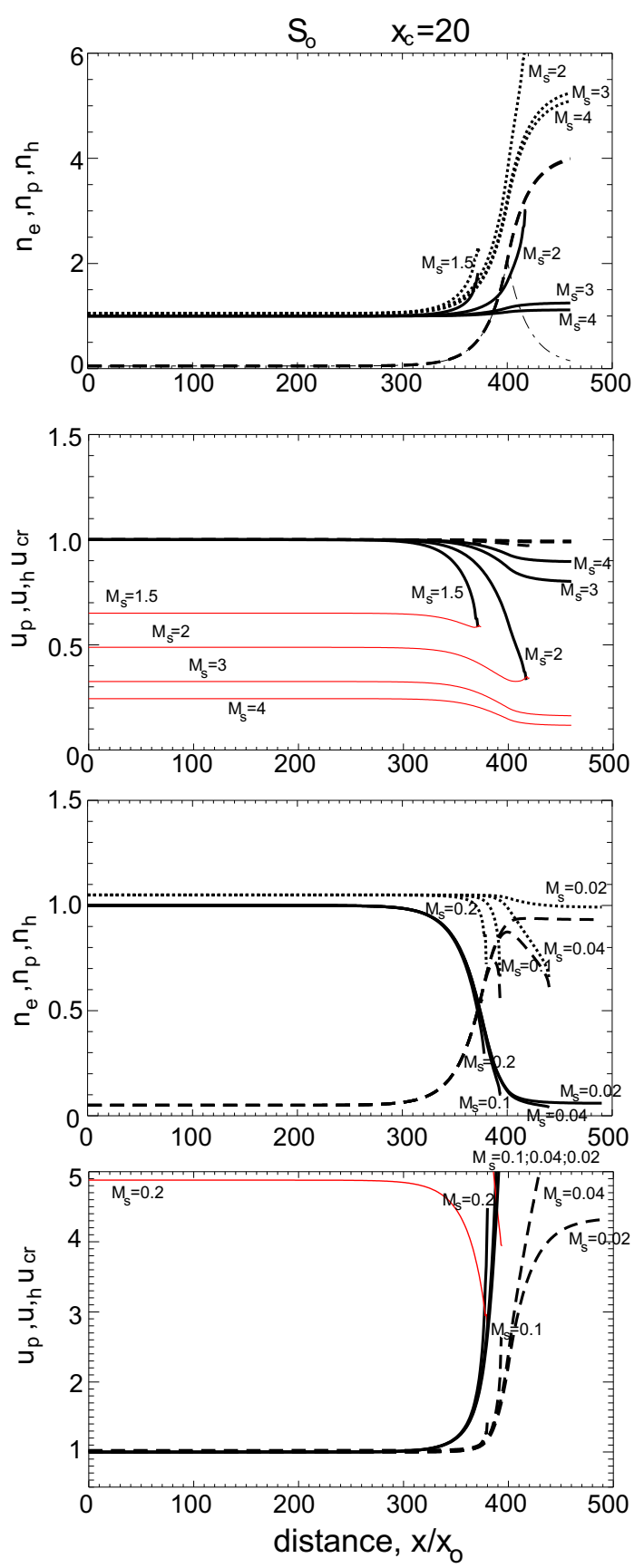

Fig. 2. Variations of the ion and electron densities (protons-the solid curves, oxygen ions - the dashed curves, electrons - the dotted curves) and velocities (protons-the solid curves, oxygen ions the dashed curves, asymptotes $\left(u_{p}=a_{p}\right)$ - the red curves) for supersonic and subsonic flows around the "ion source" (the case of cold ions). The curves are torn off at the critical points.

which shows that besides the "classical" sonic points $\left(u_{i}=c_{i}\right)$ new critical points appear, lying on the loci,

$1=\frac{a_{p}^{2}}{\left(u_{p}^{2}-c_{p}^{2}\right)}+\frac{a_{h}^{2}}{\left(u_{h}^{2}-c_{h}^{2}\right)}$.
A comparison with Eq. (8) shows that, as in a single-ion plasma where sonic and critical points coincide, in a bi-ion plasma the sonic and critical loci are also the same.

In the case of immobile heavy ions $\left(m_{h}=\infty\right)$ and cold protons, Eq. (11) becomes

$$
\frac{d u_{p}}{d x}=-\frac{u_{p} \frac{d n_{h}}{d x}}{n_{h} M_{s}^{2}\left(u_{p}^{2}-c_{s}^{2} \frac{n_{p}}{n_{e}}\right)}
$$

which allows a simple interpretation. The "supersonic", $\left(u_{p}>c_{s}\left(\frac{n_{p}}{n_{e}}\right)^{1 / 2}\right)$ ("subsonic", $u_{p}<c_{s}\left(\frac{n_{p}}{n_{e}}\right)^{1 / 2}$ ) proton flow is decelerated (accelerated) upstream of the heavy ion source, where $\frac{\partial n_{h}}{d x}>0$, and accelerated (decelerated) downstream of it (Sauer et al., 1992). Acceleration or deceleration of the proton flow is determined by the sign of the ambipolar electric field $E \sim-\frac{d n_{e}}{d x}$. In the "subsonic" regime, information about the ion obstacle propagates upstream with higher than the flow speed, and the flow undergoes the necessary changes to maintain the quasineutrality $\left(n_{e}=1 / u_{p}+n_{h}\right)$, namely, it is accelerated because of a deficit of the electrons in the heavy ion obstacle. The energy for the proton acceleration is taken from the enthalphy of the electron gas $\left(\frac{1}{M_{s}^{2}} \ln \frac{n_{e}}{n_{e o}}\right)$. In a contrast, in the supersonic case, the sign of the ambipolar electric field is reversed since the information cannot reach the flow and to maintain the charge neutrality, the electrons create their own excess in the cloud.

This picture is not greatly changed if a motion of the heavy ions is included. Figure 2 shows the spatial variations of the velocities and densities of protons, heavy ions (all ions are cold and $M_{i}=\infty$ ) and electrons in the supersonic (two upper panels) and subsonic (two bottom panels) regimes for an ion source centered at $x=400$ with a characteristic width $x_{c}=20$. Thick solid (dashed) curves correspond to the protons (heavy ions) whereas the dotted curves refer to the electrons. The dashed-dotted curve depicts the characteristics of the ion source $\left(S_{o} x_{c} \exp \left(-\left|\left(x-x_{o}\right) / x_{c}\right|\right)+\alpha\right)$. Simultaneous deceleration or acceleration of the ion species is observed. The sonic (critical) point is achieved where

$1=\frac{a_{p}^{2}}{u_{p}^{2}}+\frac{a_{h}^{2}}{u_{h}^{2}}$

Condition (15) is fulfilled on the hyperbolic sonic locus (Fig. 1b). In the region 1, the "collective" flow is "supersonic" while in the region 2, the flow is "subsonic". Red curves on the panels for the velocities (Fig. 2) show the asymptote $u_{p}=a_{p}$ of the locus. Note that the sonic (critical) point is met at different distances from the source depending on the flow parameters $\left(M_{S}\right)$. In the supersonic case, the flows at $M_{s}=3,4$ do not reach the sonic points whereas the flows with $M_{S}<2$ meet with such points in front or rear sides of the obstacle. In the subsonic case, the flow at $M_{s}=0.02$ 
does not achieve a sonic point whereas at higher Mach numbers the flows are choked at different distances.

These results can be readily interpreted. In the case of cold ions $\left(M_{p}=M_{h}=\infty\right)$ Eqs. (3) yield the energy integrals of motion:

$\frac{1}{2}\left(u_{p}^{2}-1\right)+\frac{1}{M_{s}^{2}} \ln \frac{n_{e}}{n_{e o}}=0$,

$\frac{\mu}{2}\left(u_{h}^{2}-1\right)+\frac{1}{M_{s}^{2}} \ln \frac{n_{e}}{n_{e o}}=0$,

i.e. both ions fluids are either accelerated (or decelerated) as the electron number density decreases (or increases). Variations of the electron number density are governed by the equation,

$\frac{d n_{e}}{d x}=\frac{S / u_{h}}{1-\left(\frac{a_{p}^{2}}{u_{p}^{2}}+\frac{a_{h}^{2}}{u_{h}^{2}}\right)}$.

which shows that the sign of the denominator determines the fluid structure. In the "supersonic" regime (Fig. 1b) the plus sign of the denominator implies $\frac{d n_{e}}{d x}>0$ and deceleration of the both ion fluids. In the case of "subsonic" flow, $\frac{d n_{e}}{d x}<0$ and correspondingly the ions are accelerated.

The situation becomes more complicated for finite ion temperatures. In general, the structure of a stationary onedimensional flow of a bi-ion fluid without a source is determined by the constants of motion (16), with additional terms associated with ion enthalpies (for the isothermal ions, $\frac{1}{M_{i}^{2}} \ln \frac{n_{i}}{n_{i o}}$ ). The presence of an ion source (the second term in Eq. 3d) also influences the behavior of the heavy ion flow. As a result, both ion fluids may react differently, e.g. acceleration of the protons in subfast regime (region 2 in Fig. 1a) may occur along with deceleration of the heavy ions. If then the dynamical trajectory of the collective flow crosses the "slow wave locus", corresponding to a superslow/subslow transition a "heavy ion shock" accompanied by proton rarefaction may be formed. Figure 3 shows an example of such transition. The top panel presents the distribution of the number densities of the species $(p, h, e)$. Thick solid, dashed and dotted curves correspond to the protons, heavies and electrons, respectively. The second panel depicts the velocities of the protons and heavy ions. The remarkable feature here is that the behavior of the protons and heavy ions is different, with deceleration of the heavies being accompanied by an acceleration of the protons. A sharp increase of the heavy ion density at $x \sim 330$ with a drop in the velocity $u_{h}$ is reminiscent of a shock front ${ }^{1}$. The red curve on the second panel

\footnotetext{
${ }^{1}$ It is known that the 1 -D-shocks in gases and plasmas are not stationary relative to a piston. Therefore the term "a shock" used in this paper rather corresponds to a shock-like transition which is supported only by a numerical "dissipation".
}
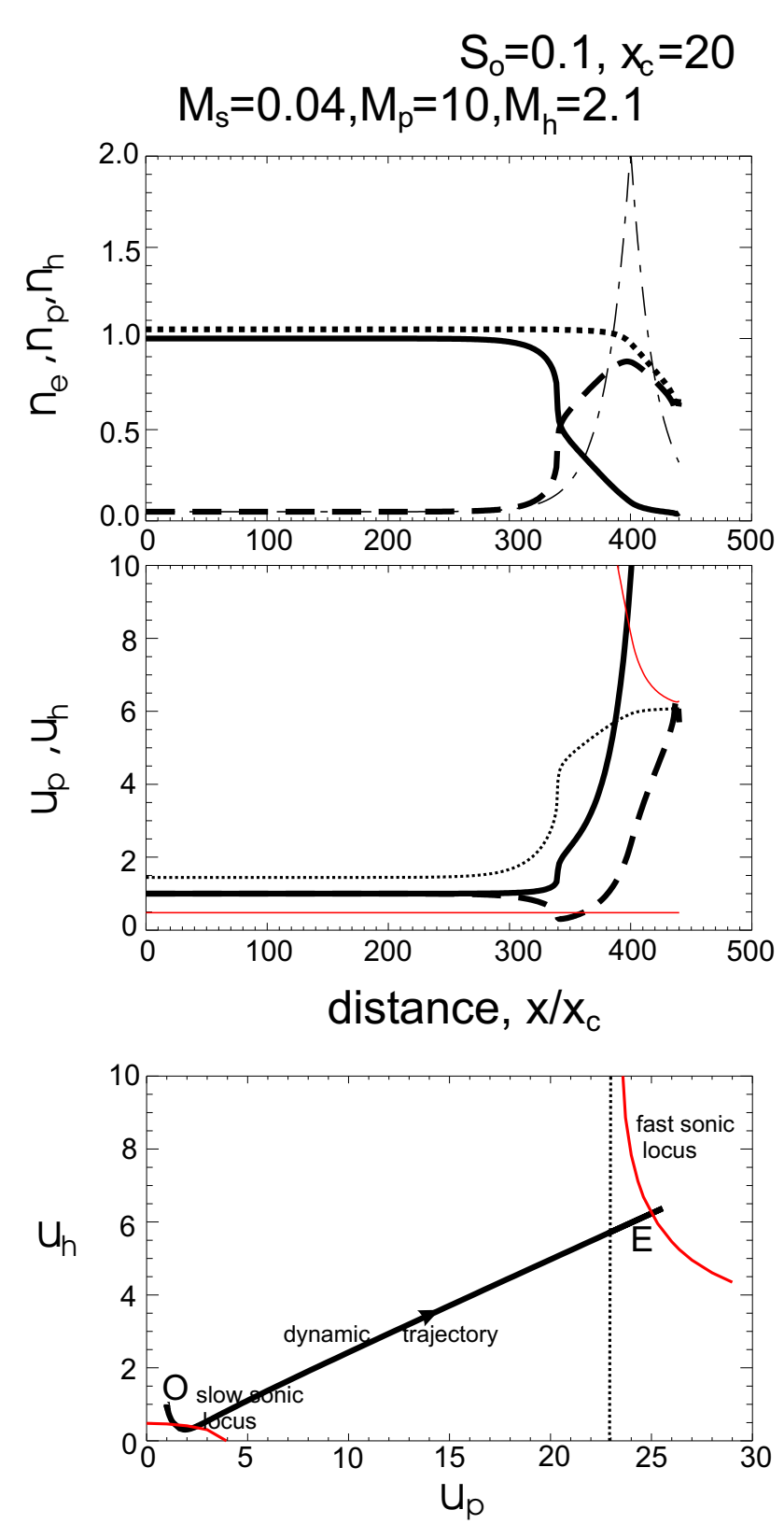

Fig. 3. Variations of the ion and electron densities (protons-the solid curves, oxygen ions - the dashed curves, electrons - the dotted curves) and velocities (the end point of the slow mode locus - the red curve) for the flow around ion source for the case of finite ion temperatures. The bottom panel shows the dynamic trajectory of the flow in the $u_{p}, u_{h}$ plane. The red curves are the slow and fast wave loci. The system starts at point "O" and goes to point "E" with crossings of the critical loci. A shock-like transition for the heavies is accompanied by the strong rarefaction of the proton flow.

gives the values of the characteristic speed which determines the end point of the slow-mode locus (see e.g. Fig. 1a). The shock position $(X \sim 335)$ corresponds to a superslow/subslow transition for the heavy ion flow that occurs close to the point 
where $u_{h}=\left(c_{h}^{2}+c_{h}^{2} /\left(1+a_{p}^{2} / c_{p}^{2}\right)\right)^{1 / 2}$ (the end point of the slow sonic locus). The dynamical trajectory $\left(u_{p}, u_{h}\right)$ is depicted by the solid curve on the bottom panel of Fig. 3 with a subsequent crossing of the slow and fast sonic loci. The system starts at the point $O$ in subfast (superslow) region, makes the transition to the subslow regime with a heavy ion shock formation and following ion acceleration. At the point $E$, the dynamical trajectory crosses another critical point on the fast mode locus and the flow is choked.

\section{Flow in the transverse magnetic field}

\subsection{Governing equations}

Here the effects of including a magnetic field transverse to the plasma flow $\boldsymbol{B}=(0,0, B)$ are considered. Faraday's law implies that $E_{y}=$ const, $E_{z}=$ const, so that $E_{y}=u_{\text {exo }} B_{z o}$, $E_{z}=0$. The electric field $E_{x}$ is determined from equation of motion for massless electrons,

$\boldsymbol{E}=-\boldsymbol{u}_{e} \times \boldsymbol{B}-\frac{\nabla p_{e}}{n_{e} e}$

The scalar product of Eq. (18) with $\boldsymbol{B}$ yields $B_{y}=0$ $\left(\nabla p_{e} \cdot \boldsymbol{B}=0\right.$ since the magnetic field is in the yz plane).

Assuming for simplicity that the ions and electrons are isothermal (the assumption of adiabatic behavior does not change the results principally), and using the dimensionless variables, we have the following momentum equations

$$
\begin{aligned}
\left(1-\frac{1}{M_{p}^{2} u_{p x}^{2}}\right) \frac{d u_{p x}}{d x}=- & \frac{1}{M_{s}^{2} n_{e} u_{p x}} \frac{d n_{e}}{d x}+\frac{\left(u_{p y}-u_{e y}\right)}{u_{p x}} B_{z}, \text { (19a) } \\
\mu\left(1-\frac{1}{M_{h}^{2} u_{h x}^{2}}\right) \frac{d u_{h x}}{d x} & =-\frac{1}{M_{s}^{2} n_{e} u_{h x}} \frac{d n_{e}}{d x}+\frac{\left(u_{h y}-u_{e y}\right)}{u_{h x}} B_{z} \\
& -\frac{\mu}{P M_{h}^{2} u_{h x}} \frac{d P}{d x},
\end{aligned}
$$

$\frac{d u_{p y}}{d x}=\frac{1}{u_{p x}}-B_{z}$

$\mu \frac{d u_{h y}}{d x}=\frac{1}{u_{h x}}-B_{z}$

which are supplemented by Ampere's law,

$$
\frac{d B_{z}}{d x}=M_{A}^{2}\left(n_{e} u_{e y}-\frac{u_{p y}}{u_{p x}}-\frac{P u_{h y}}{u_{h x}}\right)
$$

the quasineutrality condition,

$n_{e}=\frac{1}{u_{p x}}+\frac{P}{u_{h x}}$

and the current-free condition $\left(j_{x}=0\right)$, which becomes

$1+P=\frac{n_{e}}{B_{z}}$, where $M_{A}$ is the Alfven Mach number, $M_{A}=u_{o} / V_{A p}, V_{A p}$ is the Alfven speed based on the proton mass density, $V_{A p}=B_{o} /\left(\mu_{o} n_{p o} m_{p}\right)^{1 / 2}, P$ is a heavy ion flux $n_{h} u_{h x}$ (Eq. 5). The spatial variable $x$ is normalized to the length $u_{o} / \Omega_{p}$, where $\Omega_{p}=e B_{o} / m_{p}$ is the proton cyclotron frequency.

\subsection{Sonic and critical loci}

As discussed in the previous section, the loci of sonic points are determined by the dispersion equation for compressional waves in a bi-ion plasma including differential streaming by the selection of stationary waves $\left(\frac{\omega}{k}=0\right)$ in the long wavelength limit, $k \rightarrow 0$. This procedure is discussed in detail in the Appendix which shows that the equation for the sonic locus is given by

$u_{p}^{2}+\alpha \mu u_{h}^{2}=c_{p}^{2}+\alpha \mu c_{h}^{2}+(1+\alpha q) c_{s}^{2}+V_{A p}^{2}$

With $u_{p}=u_{h} \equiv V$, the value

$$
V=V_{f, b i} \equiv \frac{1}{(1+\alpha \mu)^{1 / 2}}\left(c_{p}^{2}+\alpha \mu c_{h}^{2}+c_{s}^{2}(1+\alpha q)+V_{A p}^{2}\right)^{1 / 2}
$$

yields the fast magnetosonic speed in a bi-ion plasma ( $\mathrm{Du}-$ binin et al., 2002a). Thus, in contrast to the previous case (without a magnetic field), there is only one elliptical-like locus encircling the origin, which corresponds to superfastsubfast transition (there is no a slow-wave which could give rise to a slow wave locus).

The critical loci, at which $\frac{d u_{i}}{d x}=\infty$, can be found after simple algebraic calculations from Eqs. (19), by substituting $u_{e y}$ from Eq. (20), and $\frac{d B_{z}}{d x}$ from Eq. (22) after differentiation. Consequently, we again deduce Eq. (11) with the corresponding equation for loci of critical speeds (see also Sauer et al., 1996):

$1=\frac{a_{p}^{2}+\frac{n_{p}^{2}}{n_{e}^{2}} V_{A p_{\mathrm{loc}}^{2}}}{u_{p x}^{2}-c_{p}^{2}}+\frac{a_{h}^{2}+\frac{n_{h}^{2}}{n_{e}^{2}} V_{A h_{\mathrm{loc}}}^{2}}{u_{h x}^{2}-c_{h}^{2}}$

where $V_{A p_{\text {loc }}}$ and $V_{A h_{\text {loc }}}$ are respectively the local Alfven speeds based on the local values of the magnetic field and the proton and heavy ion mass densities.

Thus, the inclusion of a transverse magnetic field implies the substitution $a_{i}^{2} \rightarrow a_{i}^{2}+\frac{n_{i}^{2}}{n_{e}^{2}} V_{A i_{\mathrm{loc}}}^{2}$ into Eq. (13) and, as in the previous case, two critical loci. Figure 4 shows examples of sonic locus (the solid curves) corresponding to superfast/subfast transition and critical loci (dotted curves) in a bi-ion plasma with $\alpha=5 \times 10^{-2}, \mu=16, \beta_{i}=0.1$ and 1 . It is observed only one point in $\left(u_{p x}, u_{h x}\right)$-space at which the sonic and critical loci intersect.

In the case of immobile heavy ions (Baumgartel and Sauer, 1992), the sonic point is achieved at

$u_{p}^{2}=c_{p}^{2}+c_{s}^{2}+V_{A p}^{2} n_{p}$ 

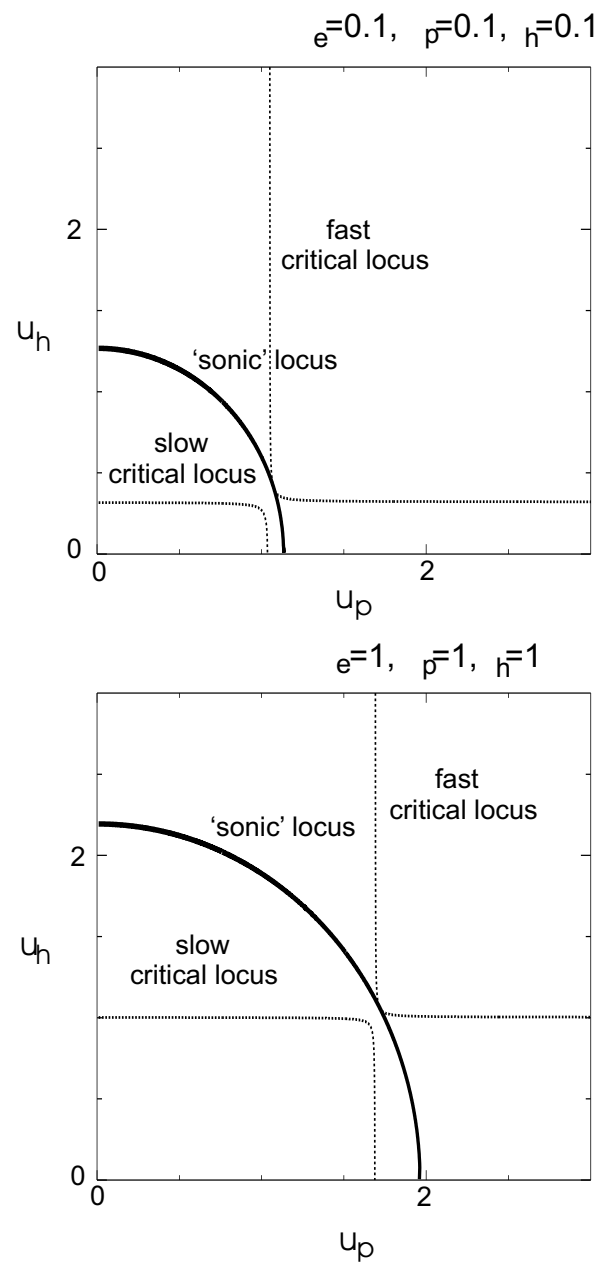

Fig. 4. Sonic (the solid curve) and critical (the dotted curves) loci for the bi-ion flows transverse to the magnetic field.

The system possesses the energy integral, namely

$\frac{1}{2}\left(u_{p x}^{2}+u_{p y}^{2}-1\right)+\frac{1}{M_{s}^{2}} \ln \frac{n_{e}}{n_{e o}}+\frac{n_{e}-1}{M_{A}^{2}}+\frac{1}{M_{p}^{2}} \ln \left(n_{p}\right)=0$,

where $n_{e o}=1+n_{h o}$. The $x$-component of the equation of motion can then be written in the form

$\frac{d u_{p x}}{d x}=f\left(u_{p x}\right)$

which elucidates the structure of the flow through the behavior of the function $f\left(u_{p x}\right)$, without numerically solving the differential equation. We will not discuss this case here but consider the general case of mobile heavy ions.

Solving Eqs. (19-20), and using Eqs. (21) and (22), yields stationary solutions for bi-ion plasma flows transverse to the magnetic field. It is observed that the magnetic field introduces a "stiffness" in the differential streaming of the ion species. In the superfast regime (sufficiently far from the critical loci) the protons and heavies stream with approximately the same speeds (Fig. 5). The small oscillations of

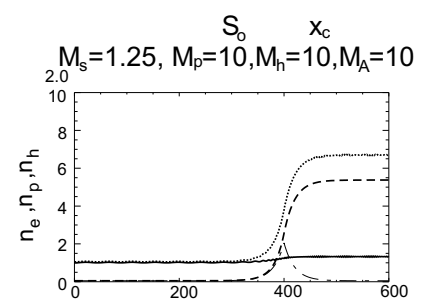

$$
\alpha=0.05, \mu=16, S_{0}=0.1, x_{c}=20 \text {, }
$$

$M_{s}=0.02, M_{p}=10, M_{h}=10, M_{A}=10$
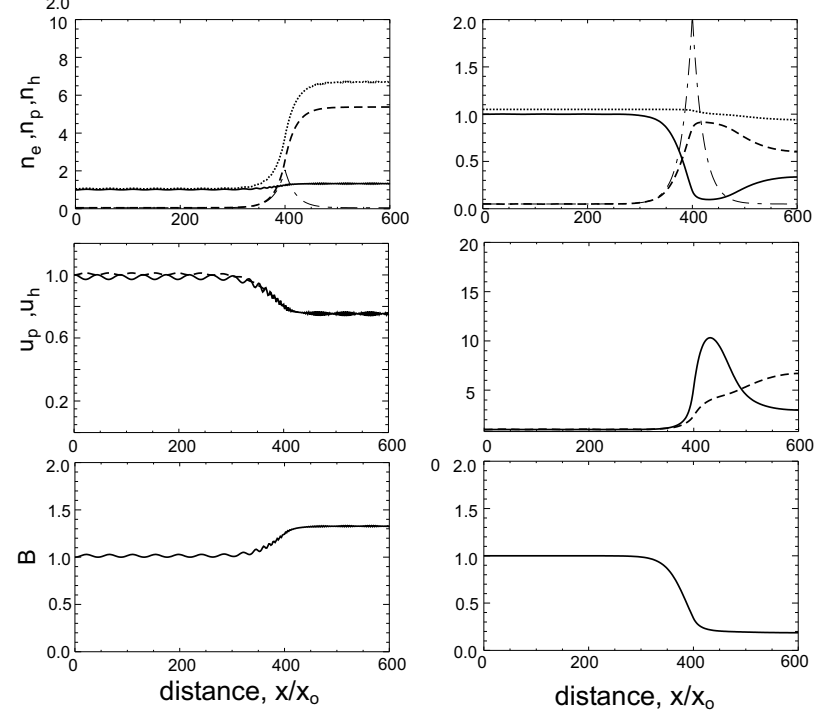

Fig. 5. Variations of the ion and electron densities, velocities and the magnetic field for the flows across the transverse magnetic field around the ion source.

the velocities correspond to the gyration of both ion fluids caused by a small difference in their initial speeds (each ion fluid feels, in their reference frame, the motional electric field due to the differential streaming and the ions begin to move on tiny cycloidal trajectories). The difference between the ion velocities increases close to the ion source. Deceleration (acceleration) of the collective plasma flow at superfast (subfast) speeds is accompanied with an increase (decrease) of the magnetic field strength. Correspondingly, a decrease (increase) of the magnetic energy at the rear of the source is balanced by an increase (decrease) of the ion kinetic energy. If a "collective" bi-ion flow reaches a critical point, lying on the critical loci, smooth solutions cannot be constructed, since they correspond to either choked or shocked flow.

Why are the "sonic" and fast critical loci different (in contrast to the magnetic field free case)? In general, dispersive effects which are naturally involved in the nonlinear Eqs. (19-20) weaken the steepening of the flow profile. As the result, the effect of overturning $\left(d u_{i x} / d x \rightarrow \infty\right)$ may occur at larger amplitudes. In magnetic field free flows the dispersive effects appear at Debye lengths. Since we have used the quasineutrality condition, these flows are nondispersive. The magnetic field introduces dispersion on much larger scales and this explains the difference between the values of the bi-ion fast magnetosonic and critical speeds. This also implies, for example, that in bi-ion flows in the range of the velocities between the fast and critical loci, one may expect the existence of solitary structures. 
a)

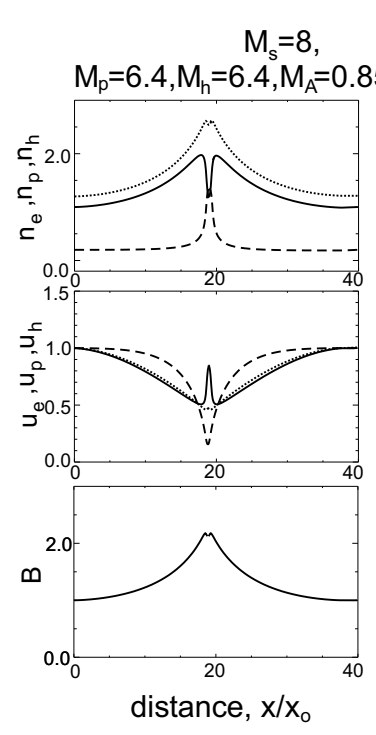

b)

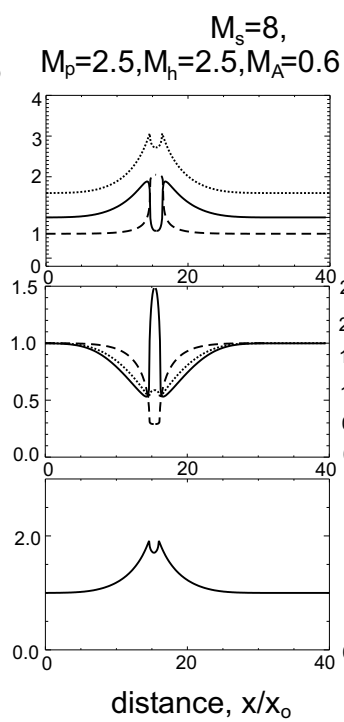

c)

$\alpha=2, \mu=16, M_{s}=8$,
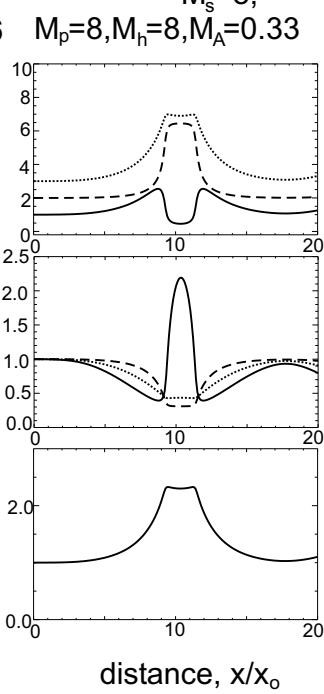

d)

$\alpha=2, \mu=16, M_{s}=8$,

$\mathrm{M}_{\mathrm{p}}=8, \mathrm{M}_{\mathrm{h}}=8, \mathrm{M}_{\mathrm{A}}=0.33$

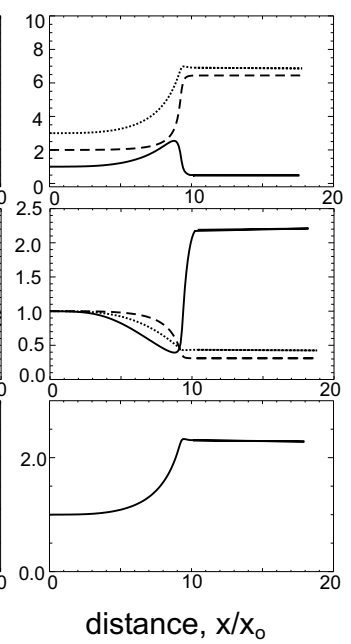

Fig. 6. Solitary structures in bi-ion plasmas with different abundance of the heavies. The right panels show a possible shock structure in a bi-ion plasma.

\subsection{Solitons}

There are specific ranges of the Mach numbers in which the protons and heavies move quite differently on the dispersive length scales. Since the dispersive length in magnetized biion plasmas is much larger than the electron and even proton inertial length a flow may be strongly affected. In fact, these flow regimes correspond to solitons propagating perpendicular to the magnetic field in multi-ion plasmas (Sauer et al., 2001, 2003; McKenzie et al., 2001; Dubinin et al., 2002a). An interesting feature of bi-ion compressive solitons is that they can contain an embedded rarefaction proton substructure. Within this rarefaction core, the protons are strongly accelerated. Figure 6 shows examples of solitons in a protonoxygen plasma with a different abundance of the heavies. A proton rarefaction core is clearly observed. Compression of the protons is followed by a sudden rarefaction and acceleration, while the oxygen ions continue to be decelerated. Such unusual behavior of different ion species hints that the inclusion of dissipation may lead to the appearance of the peculiar heavy ion shock characterized by a proton rarefaction (Fig. 6d). In particular, it has been suggested that the magnetic pile-up boundaries observed near comets and Mars could be such type of transition (see e.g. Sauer and Dubinin, 2000). Although solitons appear at sub-Alfvenic speeds (the Alfven speed is based on the proton mass density), the flow remains super-fast in the bi-ion plasma. The fast Mach number $M_{f}=u_{o} / V_{m s}\left(\right.$ where $V_{m s}=\left(V_{A p}^{2}+c_{p}^{2}+c_{s}^{2}\right)^{1 / 2}$ is the magnetosonic speed) which characterizes the plasma flow in a single-ion plasma is replaced by the value
$M_{f b i}=(1+\alpha \mu)^{1 / 2} u_{o} /\left(c_{p}^{2}+\alpha \mu c_{h}^{2}+(1+\alpha q) c_{s}^{2}+V_{A p}^{2}\right)^{1 / 2}$ in a bi-ion plasma, where $\alpha$ is the fraction of the heavies, $\alpha=n_{h o} / n_{p o}$. In the regime of soliton solutions we require $M_{f b i}>1$.

Solitary structures may be also observed in a bi-ion flow against the source. Figure 7 depicts a sequence of bi-ion solitary structures with increasing amplitude that appear when the plasma flows around a very extended source (dasheddotted curve). At $x=0$ the parameters of the collective flow lie in the range where solitons can exist. Solitons of increasing amplitude, with rarefaction of the protons in the cores are observed on approach to the centre of the source.

\section{Flow in an oblique magnetic field}

\subsection{Governing equations}

Equations (19) can be generalized to include the effects of flows oblique to magnetic field. We assume that the magnetic field at $x=-\infty$ lies in the xz-plane, $\boldsymbol{B}=\left(B_{x o}, 0, B_{z o}\right)$, where $B_{x}=$ const $=\cos \theta(\operatorname{div} \boldsymbol{B}=0), B_{z o}=\sin \theta$ in dimensionless units. For stationary flows, $E_{y}=\mathrm{const}=\sin \theta, E_{z}=0$, $E_{x}=-\frac{\sin \theta}{\cos \theta} B_{y}-\frac{1}{M_{s}^{2} n_{e}} \frac{d n_{e}}{d x}$.

Ampere's law yields,

$$
\begin{aligned}
& \frac{\partial B_{y}}{\partial x}=M_{A}^{2}\left(\frac{u_{p z}}{u_{p x}}+\frac{P u_{h z}}{u_{h x}}-n_{e} u_{e z}\right), \\
& \frac{\partial B_{z}}{\partial x}=-M_{A}^{2}\left(\frac{u_{p y}}{u_{p x}}+\frac{P u_{h y}}{u_{h x}}-n_{e} u_{e y}\right),
\end{aligned}
$$


in which the electron velocity is given by the Eq. (18),

$u_{e y}=\frac{1+P}{n_{e}} \frac{B_{y}}{\cos \theta}, u_{e z}=\frac{1+P}{n_{e}} \frac{B_{z}}{\cos \theta}-\frac{\sin \theta}{\cos \theta}$.

The current-free condition $u_{e x}=(1+P) / n_{e}$ yields the $x$ component of the electron speed. Thus we have nine nonlinear differential equations for nine variables $u_{p x}, u_{p y}, u_{p z}, u_{h x}, u_{h y}, u_{h z}, B_{y}, B_{z}, n_{e}$.

\subsection{Sonic and critical loci}

The results of Sect. 3.2 and the appendix are readily generalized to the case of the oblique magnetic field although the number of wave modes significantly increases. These modes can give rise to several "sonic" loci which, in general, do not coincide with the critical loci.

In contrast to the case of flows transverse to the magnetic field, the number of constants of motion for oblique propagation is less than is necessary to obtain algebraic relations between the variables contained on the RHS of the motion equations for $d u_{i x} / d x$. Since, in general, terms on the RHS may be also proportional to $d u_{i x} / d x$, the equation for critical loci cannot as readily be obtained as in the transverse case. However, the problem of critical points can be considered from another viewpoint. It has been shown (Dubinin et al., 2003b; McKenzie et al., 2004) that stationary one-dimensional and adiabatic flows oblique to the magnetic field, possess the following energy integrals

$m_{i}\left(\frac{\boldsymbol{u}_{i}^{2}}{2}+\frac{\left(\boldsymbol{E} \times \boldsymbol{u}_{i}\right)_{x}}{B_{x}}\right)+w_{i}+q w_{e}=\mathrm{const}, \quad i=p, h$

where $w_{i}$ and $w_{e}$ are, respectively, the ion and electron enthalpies,

$w_{i, e}=\frac{\gamma_{i, e}}{\gamma_{i, e}-1} \frac{p_{i, e}}{n_{i, e}}$.

These relations imply that at any point of the flow the change in energy density per unit charge of any ion species is accompanied by an equal and opposite change in the electron energy density. In dimensionless units, the energy integrals for each ion species can be written in the form

$$
\begin{aligned}
u_{i x}^{2}+u_{i y}^{2}+\left(u_{i z}+\frac{E_{y}}{B_{x}}\right)^{2} & =1+\frac{E_{y}^{2}}{B_{x}^{2}}+\frac{2}{M_{i}^{2}} \ln \frac{1}{u_{i x}} \\
& +\frac{2}{m_{i} M_{s}^{2}} \ln \frac{1}{u_{e x}},
\end{aligned}
$$

where $i=p, h, m_{i}=1, \mu$. Here we have assumed isothermal electrons and ions $\left(w_{i}=\frac{m_{i}}{M_{i}^{2}} \ln n_{i}\right)$ and the constants are fixed from the conditions at $x=-\infty$.

For cold electrons, Eqs. (33) for the protons and heavies decouple, which leads to the following simple geometrical interpretation. The tip of the vectors $\boldsymbol{u}_{i}$ move on the surfaces of two spheroids with centers at $\left(0,0,-E_{y} / B_{x}\right)$ which cross the initial point $O$
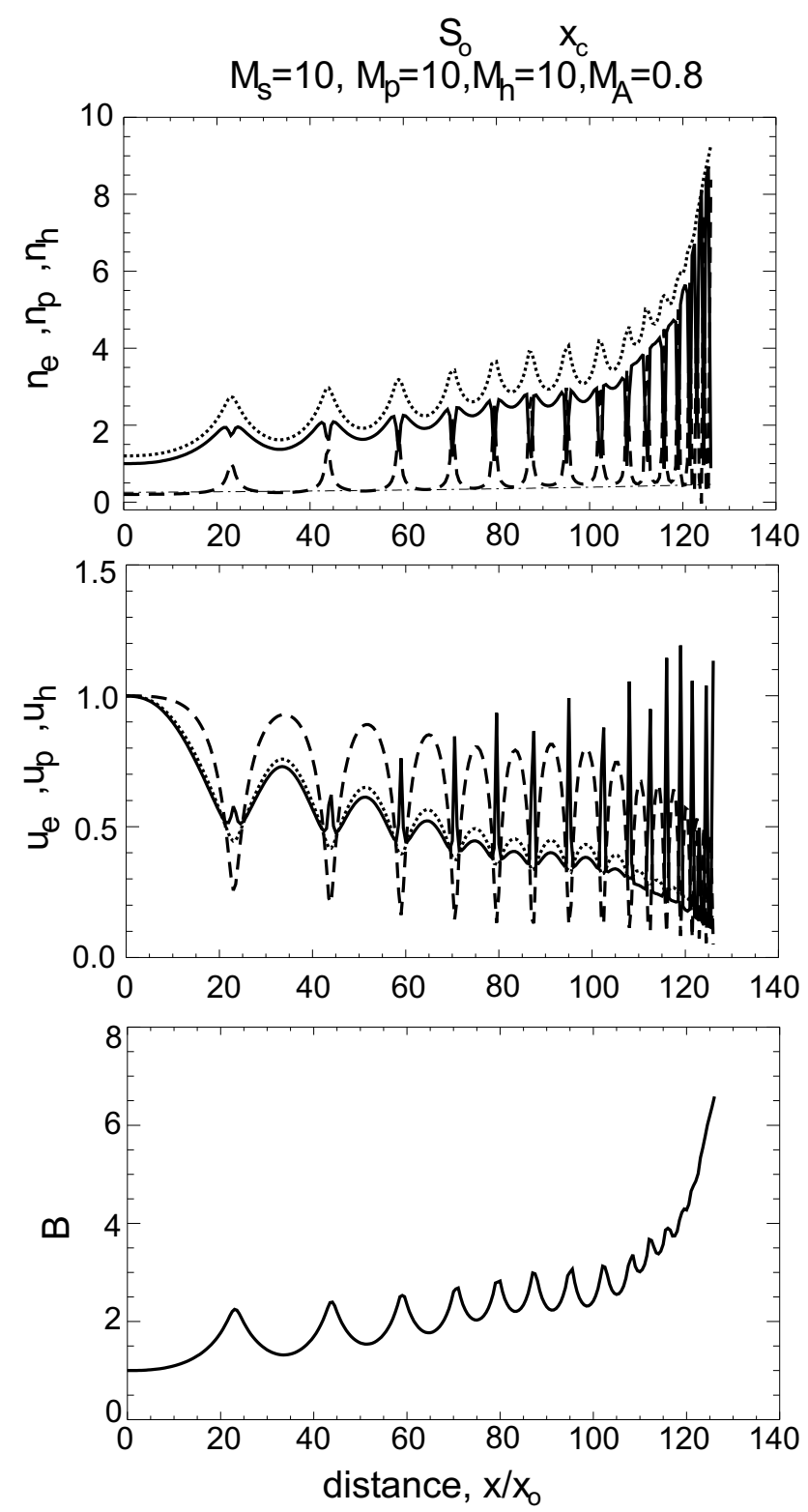

Fig. 7. A train of solitatary structures arising from the plasma flow around very extended source.

$(1,0,0)$. Cross-sections of the surfaces at $u_{i x}=$ const are the circles with the radii $R_{i}=\left(E_{y}^{2} / B_{x}^{2}-f\left(u_{i}\right)\right)^{1 / 2}$, where $f\left(u_{i}\right)=\left(u_{i x}^{2}-1\right)+\frac{2}{M_{i}^{2}} \ln \left(1 / u_{i x}\right)$ are the Bernoulli energy functions for the longitudinal flows (the sum of changes in the kinetic energies and enthalpies). It is important to note that the radii of the cross-sections depend only on the longitudinal speed $u_{i x}$. Figure 8, which depicts examples of Bernoulli functions and spheroids, and shows that for supersonic flows $\left(M_{i}>1\right)$ accessible rarefactive flows are restricted by the top of the spheroids (the point $R$ on $f_{i}$ and on the spheroid, where the point $O$ corresponds to the initial 

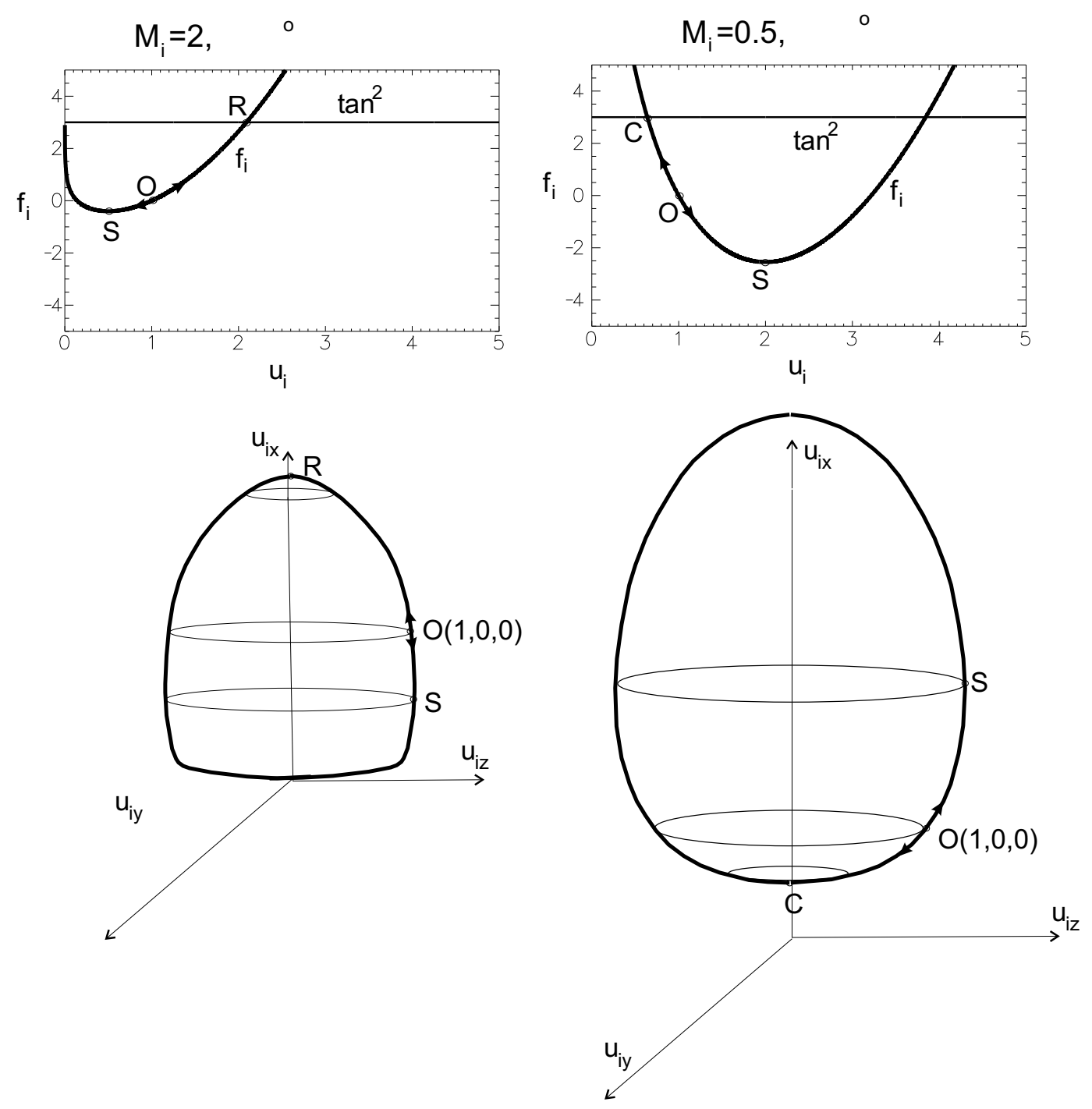

Fig. 8. Examples of Bernoulli functions and spheroids for subsonic and supersonic flows with cold electrons. The system starts at point "O" and moves along rarefactive or compressive paths.

state). Compressive flows are restricted (critical points) by the position of "sonic" points $\left(M_{i}=1\right)$ which correspond to minima $(S)$ of the functions $f\left(u_{i}\right)$. In the subsonic regime $\left(M_{i}<1\right)$, a possible compression is limited by the bottom of the spheroids (point $C$ ), while an accessible rarefaction is limited by the "sonic" point $S$ at $u_{i x}=1 / M_{i}$ (minimum of $\left.f\left(u_{i}\right)\right)$.

When the electron pressure is taken into account, the radius of the cross-section of the spheroid for the protons (heavies) depends not only on the constants $E_{y}, B_{x}$ and $M_{i}$, but also on $M_{s}$ and, more importantly, the value of the local heavy ion (proton) speed $u_{h x}\left(u_{p x}\right)$. The generalized Bernoulli functions which determine the shape of the spheroids then become,

$$
\begin{aligned}
f_{i} & =\left(u_{i x}^{2}-1\right)+\frac{2}{M_{i}^{2}} \ln \frac{1}{u_{i x}}+\frac{2}{m_{i} M_{s}^{2}} \ln \left(\frac{1}{u_{p x}}+\frac{\alpha}{u_{h x}}\right) \\
& -\frac{2}{m_{i} M_{s}^{2}} \ln (1+\alpha q)
\end{aligned}
$$

If $\alpha u_{p x} \ll u_{h x}$, then $f\left(u_{p}\right) \approx\left(u_{p x}^{2}-1\right)+2\left(1 / M_{p}^{2}+1 / M_{s}^{2}\right) \ln$ $\left(1 / u_{p x}\right)$ and the critical speed for the protons is $\left(c_{p}^{2}+c_{s}^{2}\right)^{1 / 2}$.

Figure 9a shows the Bernoulli function for the bi-ion flows $\left(\alpha=0.05, \mu=16, \theta=60^{\circ}\right)$ at $M_{i}=1.48, \quad M_{s}=\infty$ and $M_{S}=1.48$, which corresponds to flows with $M_{A}=1.48$ in a plasma with $\beta_{i}=1$. In the case of cold electrons $\left(M_{s}=\infty\right)$, a sonic point is reached at $u_{i x} \sim 0.7$. Since $M_{p}=M_{h}$ the shapes of both spheroids are the same, although the trajectories of the vectors $\boldsymbol{u}_{i}$ on their surfaces may be very different, e.g. 

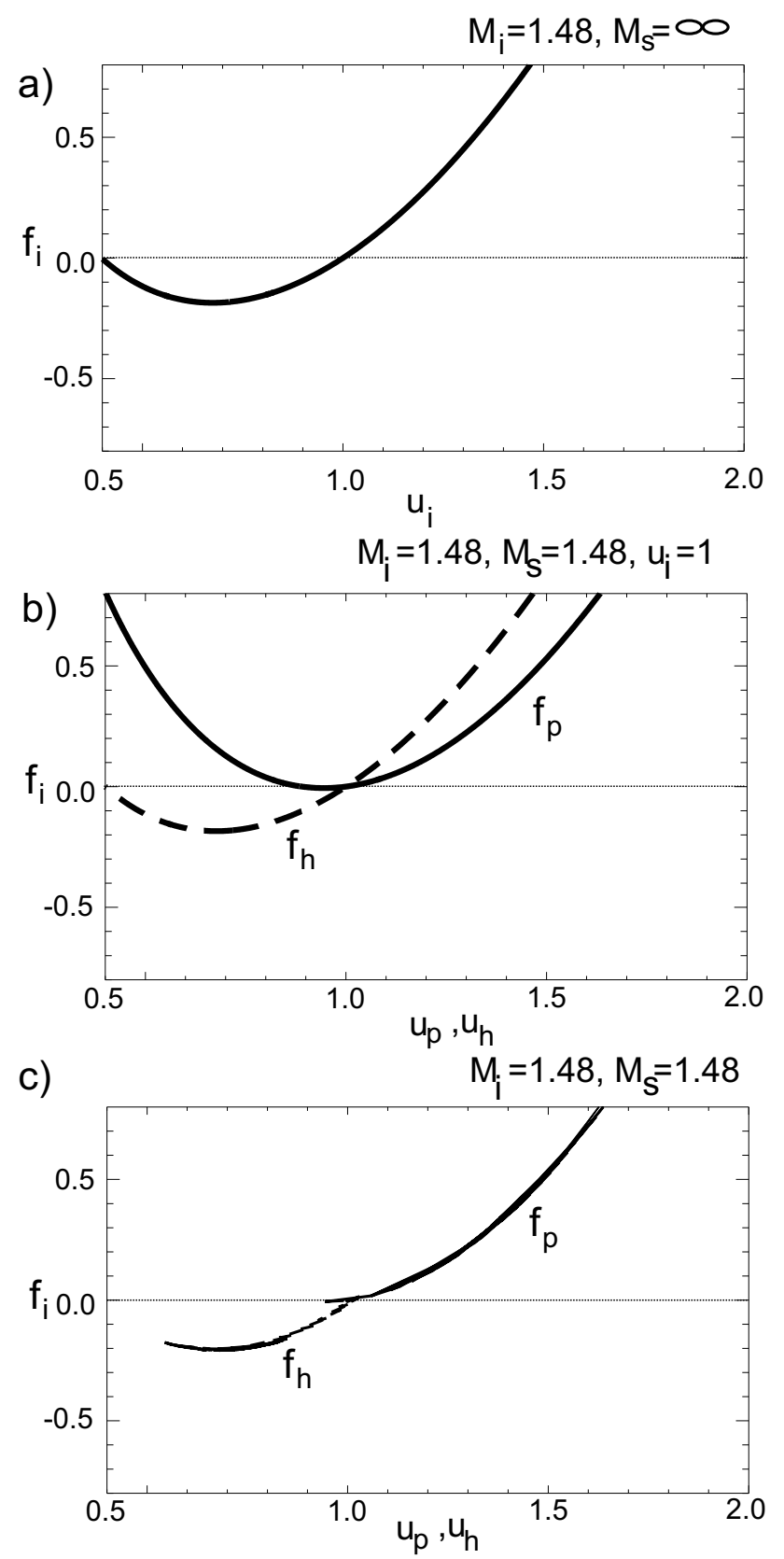

Fig. 9. Examples of Bernoulli functions for bi-ion flows with warm electrons.

acceleration of the protons along with deceleration of the heavies and vice versa. However, in both flows, sonic points are achieved at $u_{i x}=1 / M_{i}$. Therefore it is possible that the flow of only one ion fluid becomes sonic. When electron pressure is included, the motion of the ions becomes more complicated. The Bernoulli functions $f_{p}$ and $f_{h}$ are now different. Figure $9 \mathrm{~b}$ shows $f_{p}$ and $f_{h}$ (Eq. 34) for $M_{s}=1.48$ (here we assume also for a simplicity that $u_{h x}=1\left(u_{p x}=1\right)$ for $f_{p}\left(f_{h}\right)$ ). The sonic point of the proton flow is reached at $u_{p x} \sim 0.95$, i.e. accessible smooth compressive proton flows

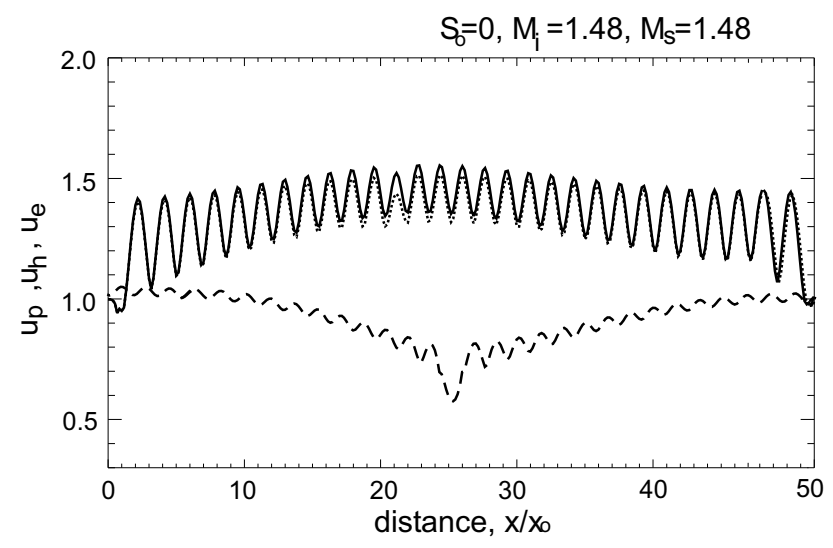

Fig. 10. Coherent solitary-oscillatory structure corresponding to the upper "window" in Fig. 13. Despite of strong oscillations the vectors $\boldsymbol{u}_{i}$ move on the smooth surface of the Bernoulli spheroid (Fig. 9c).

are extremely restricted, while the sonic point for the heavies remains approximately the same $\left(u_{h x} \sim 0.7\right)$. Figure $9 \mathrm{c}$ shows the functions $f_{p}$ and $f_{h}$ for stationary solutions for $u_{p x}$ and $u_{h x}$ by solving the full set of nonlinear differential equations for the bi-ion flow with $S=0$. The agreement between the curves on Figs. $9 \mathrm{~b}$ and $\mathrm{c}$ is reasonable although the motion of the ions is very different and complicated as is observed in Fig. 10 which depicts the exact stationary solutions. The ion motion exhibits strong oscillations, although the tips of the vectors $\boldsymbol{u}_{i}$ move on the smooth surface of the Bernoulli spheroids with the radii determined from the Bernoulli functions shown in Fig. 9c. In this case, a coherent motion of both fluids is maintained by a perfect coupling between the ions (a solitary-like structure).

In general, the Bernoulli spheroids can become strongly contracted or expanded during the collective motion since the motion of the protons (heavies) influences the Bernoulli functions of the heavies (protons), $f_{h}\left(f_{p}\right)$ in a rather complicated way. The top panel of Fig. 11 shows an example of how the radii $R_{i}$ of the cross-sections of the proton (heavy ion) spheroids at $u_{i x}=$ const vary in the bi-ion flow against the extended ion source. Lower panels show the number densities of the species, the longitudinal speeds and the magnetic field. The flow consists of many perturbations which provide rapid expansions and contractions of the spheroids (the different radii $R_{p}\left(R_{h}\right)$ are observed for the same values of $u_{p x}$ $\left(u_{h x}\right)$ but different values of $u_{h x}\left(u_{p x}\right)$ ).

The geometrical interpretation of the energy integrals (33) shows that differential ion streaming of the collective flows oblique to the magnetic field is more easily accessible than in the transverse case. Both ion fluids may respond differently on an "ion obstacle". As a result, the flow structures are more richly varied. Figure 12a depicts the formation of a "heavy ion shock" when the velocity of the heavies crosses the slow critical locus. At this transition, the protons are however 

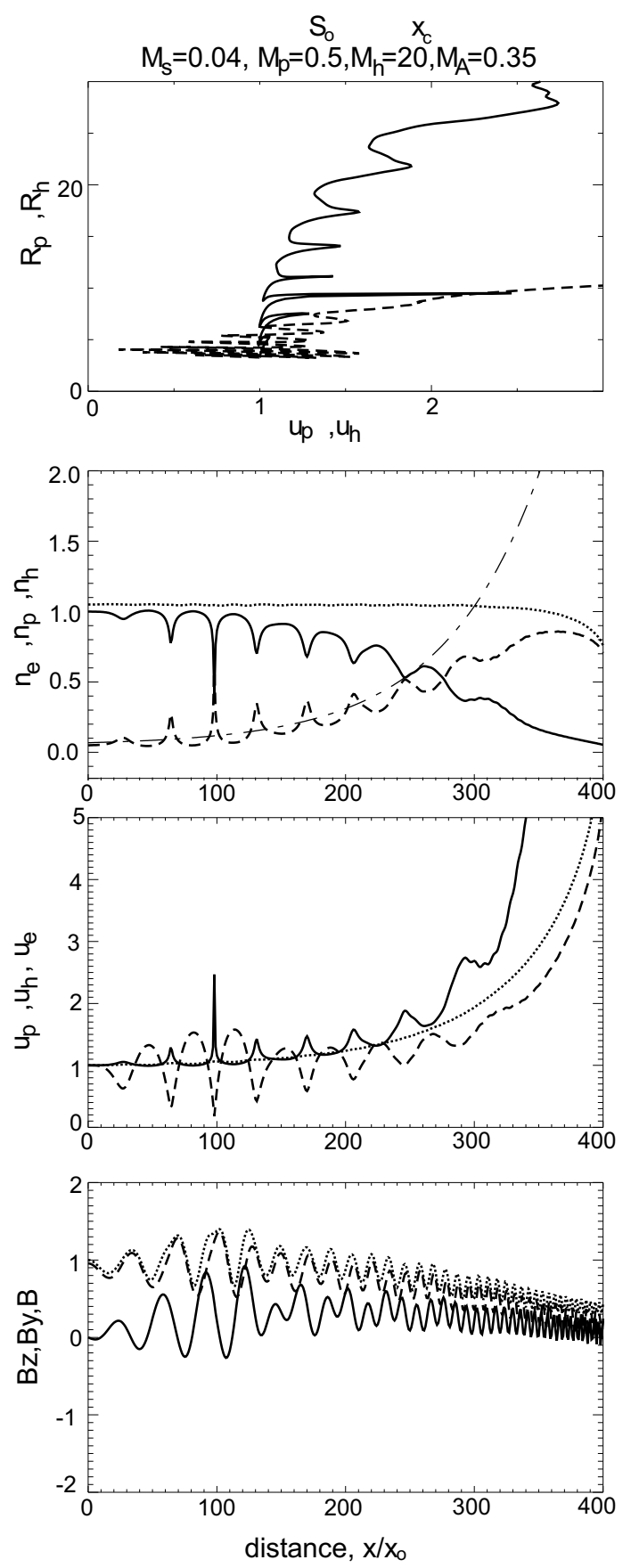

Fig. 11. (top panel) Variations of the radii $R_{i}$ of the cross-sections of the proton (heavy ion) spheroids with the longitudinal speeds $u_{i x}$. The value of $R_{p}\left(R_{h}\right)$ depends not only the constants $E_{y}, B_{x}, M_{i}$, but also on the value $u_{h x}\left(u_{p x}\right)$ at this point. (lower panels) Variations of the ion and electron number densities, velocities and the magnetic field for the bi-ion flow around the extended ion cloud.

strongly accelerated. Figure $12 \mathrm{~b}$ shows an example of how the velocity of the heavies exhibits large-amplitude oscillations followed by a shock-like transition. As in the previous case, the heavy ion shock is accompanied by a proton rar- efaction. Figures 12c, d present examples of compressive and rarefactive large-amplitude waves of growing amplitude on approaching the obstacle.

The functions $f_{p}$ and $f_{h}$ cannot always be considered as the Bernoulli functions where minima determine the critical points. For example, when the term corresponding to the electron enthalphy dominates over the ion kinetic and thermal energies, the behavior of $f_{i}$ is very different from a "classical" Bernoulli function, and only when $u_{i x}$ (kinetic energy) increases, the function $f_{i}$ exhibits a minimum at the sonic point.

Assuming that the transverse ion velocities $u_{i y}, u_{i z}$ (the magnetic field components $B_{y}, B_{z}$ can be expressed as functions of the transverse speeds using the momentum flux conservation laws) are not proportional to $d u_{i x} / d x$, and performing simple algebraic manipulations with the governing equations, yields $\frac{d n_{e}}{d x}=\frac{N_{e}}{D_{e}}$, where $D_{e}$ is a function only of the longitudinal velocities $u_{i x}$. The condition $D_{e}=0$ for the critical points reduces to the same expression (13) for the magnetic field-free case, namely

$1=\frac{a_{p}^{2}}{u_{p x}^{2}-c_{p}^{2}}+\frac{a_{h}^{2}}{u_{h x}^{2}-c_{h}^{2}}$

which does not explicitly contain the magnetic field, although the behavior of $u_{p x}$ and $u_{h x}$ is, in fact, controlled by the magnetic field.

\subsection{Solitary and oscillatory structures}

It has been shown (Sauer et al., 2001, 2003; Dubinin et al., 2002b, 2003b) that bi-ion stationary flows with speeds in the "windows" between the longwavelength limits $(k=0)$ of the phase speeds of the linear wave modes, can generate solitary or oscillatory structures. Figure 13 presents the phase speed of waves in a bi-ion plasma $\left(\alpha=0.05, \beta_{i}=1, \mu=16, \theta=60^{\circ}\right)$. Dashed areas depict the ranges of the velocities at which solitary (or oscillatory) wave structures can be observed in a bi-ion plasma with $\alpha=0.05, \beta_{i}=1, \mu=16, \theta=60^{\circ}$. Figure 10 has already provided us with an example of a coherent stationary structure in a bi-ion flow when the flow speed (at $x=-\infty$ ) lies in the upper "window" in Fig. 13. The existence of a phase velocity minimum on the upper resonance mode gives rise to oscillations embedded within the solitary structure. This type of solitary waves arises from a specific feature, namely the existence of an extremum on the curve of the phase speed $\omega / k(k)$ (Sauer et al., 2001, 2003; Dubinin et al., 2002b, $2003 \mathrm{a}, \mathrm{b})$. Since the minimum is rather smooth the structure differs from a typical oscilliton.

The strongly nonlinear wavy structure shown in Fig. 14 provides another example of a stationary flow when $u_{i x o}=0.434$, i.e. the speed occurs in the second window for oscillitons (Fig. 13). At larger wave speeds, the amplitude of the oscilliton increases and the expanding protons can reach 

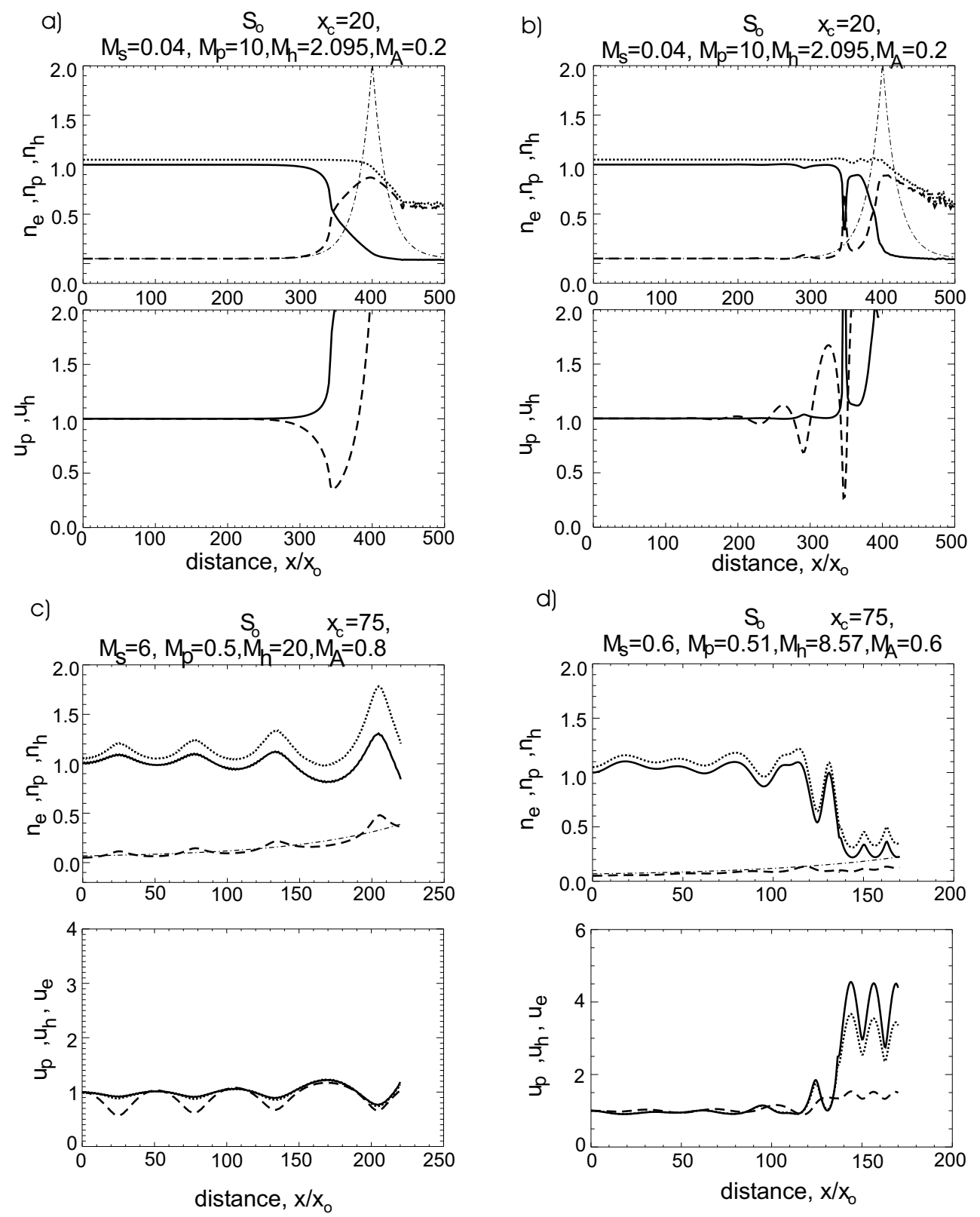

Fig. 12. Examples of the structures observed for the collective ion flows obliquely to the magnetic field.

the critical point corresponding to the minimum of $f_{p}$ with the result that a smooth solution cannot be constructed. At speeds below $0.37 V_{A p}$, but above the velocity of the "heavy ion" mode $\left(0.31 V_{A p}\right)$ classical solitary waves can exist.

The inclusion of an ion source naturally disturbs a soliton (oscilliton) structure. However, because of the rather large characteristic width of bi-ion oblique solitons their typical features can be readily recognized within the large-scale flow patterns. Figure 15a presents an example of the solitary wave. In contrast to classical solitons in a single-ion plasma, their structures in a bi-ion plasma are much richer.
For example, here we observe a different behavior of the different ions with a small reacceleration of the heavies in the centre of their compression. The magnetic field component $B_{z}$, besides the main maximum, reveals two clear "shoulders" at the edges. It is interesting that all these features can be readily recognized when the ion source is placed at $x=400$ (Fig. 15b). The structure observed closer to the source $(x \sim 360)$ is reminiscent of the shock-like transition in Fig. 3 for the magnetic field free case while a part of an oblique soliton is clearly displayed far upstream of the obstacle. 


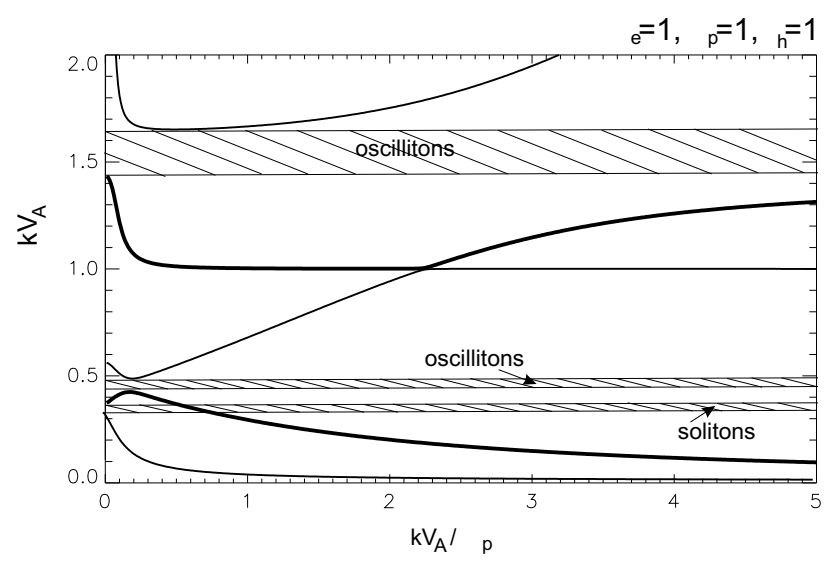

Fig. 13. The dispersive diagram $(\omega / k-k)$ for oblique propagating waves in a bi-ion plasma. In windows between the longwavelength limits $(k=0)$ of the phase speeds solitary and oscillatory waves exist.

The shock transition occurs with a sudden jump (drop) in the heavy ion (proton) density. At this point, $\frac{d n_{e}}{d x}=\rightarrow \infty$. Note that the functions $f_{p}$ and $f_{h}$ have no clear minima at this point and therefore cannot be considered as classical Bernoulli functions. In contrast, at greater distances $(x \sim 440)$, where the protons and heavies are strongly accelerated (and where contribution of the kinetic energy becomes essential) the collective flow reaches the critical point where $D_{e} \rightarrow 0$ and also $f_{p, h}$ reach their minima.

\section{Conclusions}

This paper explores in detail the features of stationary flows in bi-ion plasmas. One of the most important points is the identification of critical loci, on which the flow is shocked or choked, with the idea of sonic loci providing a generalization of the concept of a sonic point to multi-ion flows with differential ion streaming. In this sense the dispersion equation for stationary waves, as viewed in a lab frame in which differential ion streaming takes place, yields the sonic loci in $\left(u_{p}, u_{h}\right)$ space which is therefore subdivided into various regions, as to the nature of the flow, superfast, subfast, superslow and subslow. These ideas are extended to include the effects on the flows of the magnetic field. The flows set up in response to an ion source are studied within this framework. These can reveal rather complicated structures, depending upon the regimes into which the unperturbed flow speed finds itself. For example, it is possible that "heavy ion shocks" are accompanied by a proton rarefaction. This feature is already present in magnetic field free flows of a bi-ion plasma with finite ion temperatures. Two critical loci also appear in the case of flow in the transverse magnetic field. A different behavior of the ion fluids is revealed in the specific range of the velocities where bi-ion solitary structures can be generated.
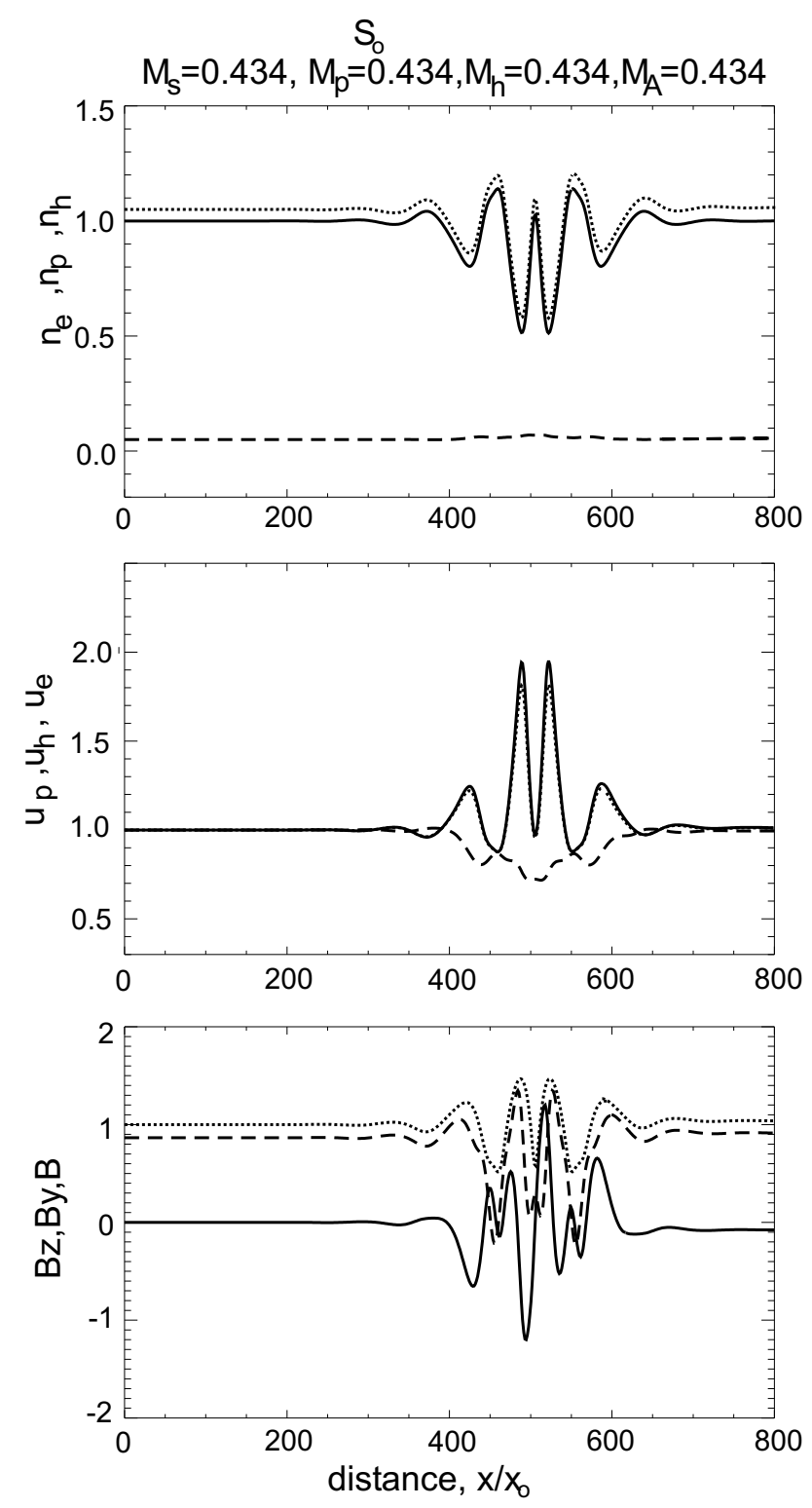

Fig. 14. Oscillatory structure in bi-ion flow without a source.

Such solitons exhibit the remarkable property of a heavy ion deceleration accompanied by a rather sharp proton rarefaction near the centre of the wave, with concomitant features in the magnetic field profile. Inclusion of dissipation may lead to shock transitions characterized by the deceleration (acceleration) of the heavies (protons). In other velocity regimes the magnetic field introduces a stiffness in the differential streaming of the ion fluids. Magnetic obliquity significantly increases the variety of accessible collective ion flows with differential ion streaming. The analysis of such flows and critical points for an oblique case is facilitated by the use of energy integrals and properties of the Bernoulli energy functions for the longitudinal ion speeds. An extremely useful geometrical interpretation of the integrals of motion elucidates 
a)
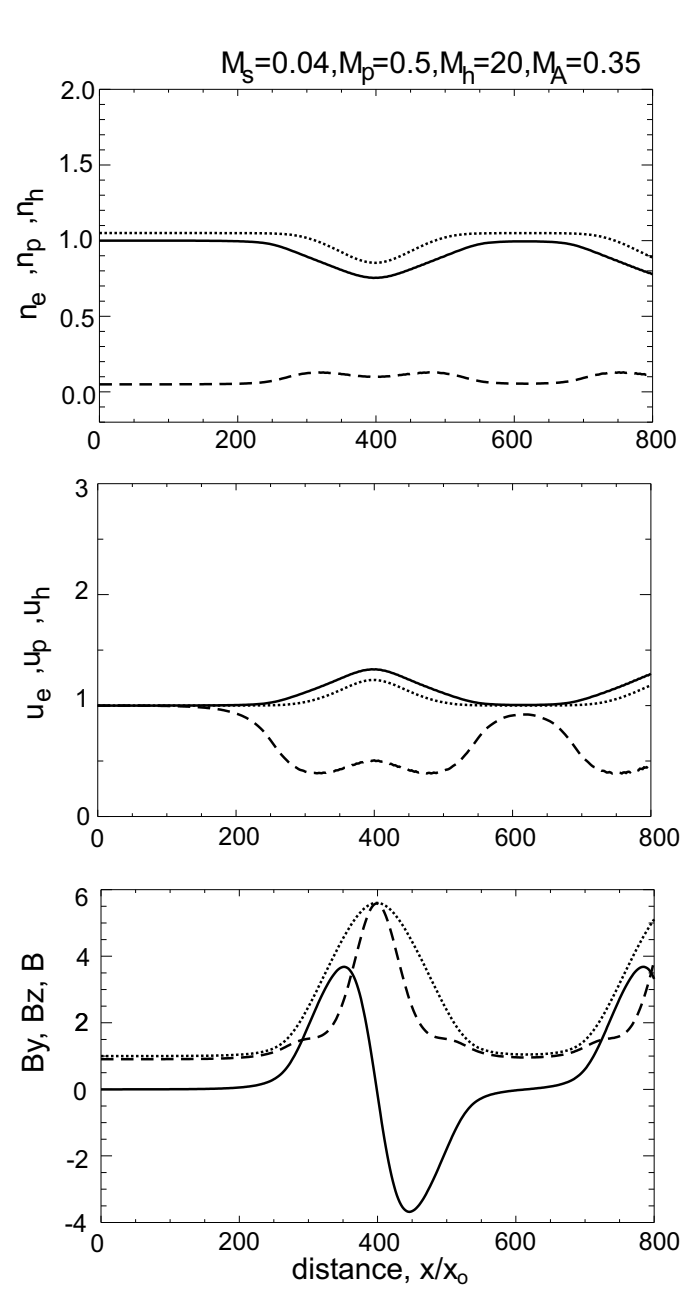

b)
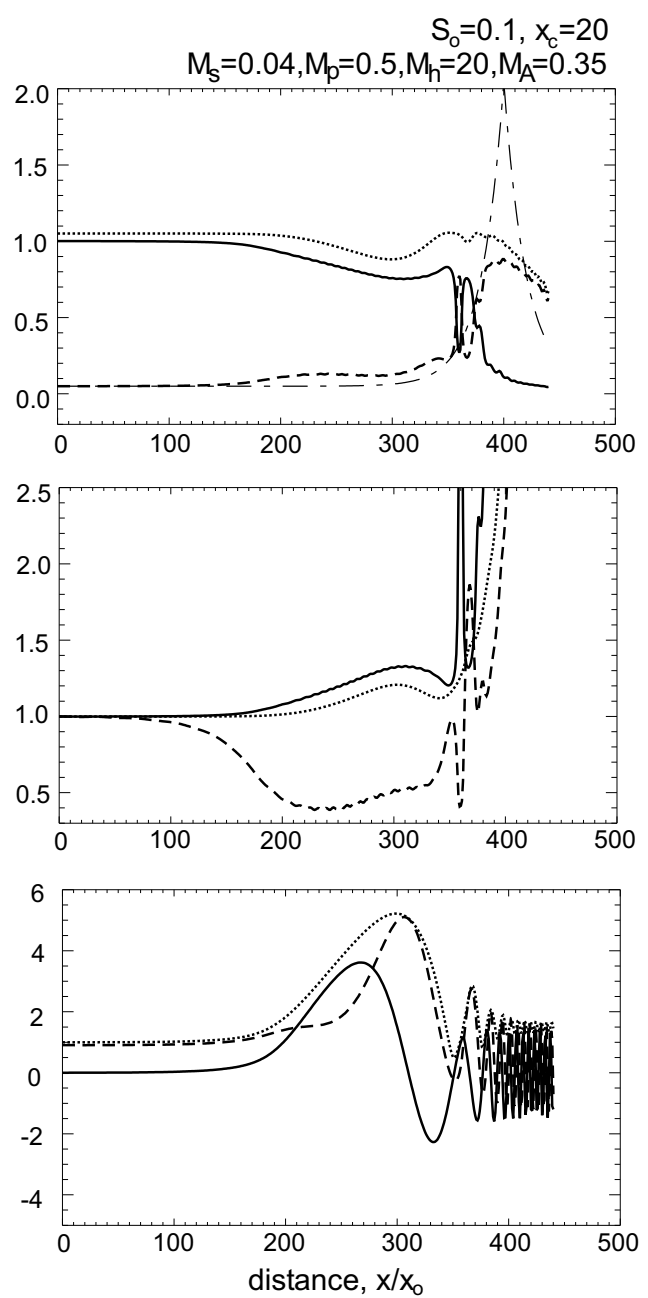

Fig. 15. (a) Solitary structure in bi-ion flow (without a source) propagating obliquely to the magnetic field, (b) "Perturbations" in solitary structure by the ion source. Shock-like transitions for the heavies accompanied by the proton expansion appear at critical points.

the main characteristic features of the collective ion dynamics of a multi-ion plasmas. The results are underlined by a series of numerical solutions which illustrate the rich, complex flow structures which arise in various flow regimes in response to various ion sources. In particular, heavy ion shocks accompanied by the proton rarefaction may appear for the flows oblique to the magnetic field. Coherent wave motions, associated with very specific features of solitary structures in a bi-ion plasma, can also be readily generated. Since the characteristic width of oblique solitons (oscillitons) can reach several hundreds of the proton inertial lengths such structures are easily recognizable.

\section{Appendix A}

\section{Dispersion of waves propagating transverse to the magnetic field in a bi-ion plasma}

For plane wave disturbances varying as $\exp (i k x-i \omega t)$ the linearized equation of motion for ions in dimensionless variables can be written in the $2 \times 2$ matrix form,

$$
\hat{L}_{i} \boldsymbol{u}_{i}=\boldsymbol{E}
$$

Here $\hat{L}_{i}$ is the matrix

$L_{i}^{j k}=\left|\begin{array}{ll}{\left[-i\left(\omega-k V_{i}\right) m_{i} / q_{i}+\frac{i m_{i} k^{2} \beta_{i}}{\left(\omega-k V_{i}\right) q_{i}}\right]} & -1 \\ \frac{\omega}{\omega-k V_{i}} & -\frac{i \omega m_{i}}{q_{i}}\end{array}\right|$,

where $\boldsymbol{u}_{i}=\left(u_{i x}, u_{i y}\right), \boldsymbol{E}=\left(E_{x}, E_{y}\right)$ are the velocity and electric field perturbations, $i=p, h, m_{i}$ and $q_{i}$ are respectively the 
mass and charge of the ions $\left(m_{p}=1, m_{h}=\mu, q_{p}=1, q_{h}=q\right)$, $\beta_{i}=c_{i}^{2} / V_{A p}^{2}$ (here $c_{i}$ are the thermal speeds, $c_{i}^{2}=T_{i} / m_{i}$ and $V_{A p}$ is the Alfven speed based on the proton mass density). We assume that the ions are isothermal and have bulk speeds $V_{i}$ along the x-axis, while the magnetic field is parallel to the z-axis $\left(B_{x}=0, \quad B_{y}=0, B_{z o}=1\right)$. Faraday's law yields the magnetic field perturbation $b_{z}=k E_{y} / \omega$. The continuity equations give the density perturbations $n_{i}=\frac{\alpha_{i} k u_{i x}}{\omega-k V_{i}}$, where $\alpha_{i}=n_{i o} / n_{p o}\left(\alpha_{p}=1, \alpha_{h}=\alpha\right)$.

Time and space variables ( $t$ and $x$ ) are normalized to $\Omega_{p}^{-1}$ and $V_{A p} / \Omega_{p}$, respectively, where $\Omega_{p}$ is the proton gyrofrequency. The velocities are normalized to the Alfven speed $V_{A p}$ and the electric field to the value $E_{o}=V_{A p} B_{o}$.

Correspondingly, for massless electrons $\hat{L}_{e} \boldsymbol{u}_{e}=\boldsymbol{E}$, where

$L_{e}^{j k}=\left|\begin{array}{cc}-\frac{i k^{2} \beta_{e}}{\left(\omega-k V_{i}\right) q_{i}} & -1 \\ \frac{\omega}{\omega-k V_{e}} & 0\end{array}\right|$,

in which we have used $n_{e}=(1+\alpha q) \frac{k u_{e x}}{\omega-k V_{e}}$. Note that Eqs. (36) could be reduced to matrixes of the second order, since $E_{z}=0$ which follows from the $z$-component of the electron equation of motion.

Maxwell equations $\operatorname{curl} \boldsymbol{E}=-\frac{\partial \boldsymbol{B}}{\partial t}$ and $\operatorname{curl} \boldsymbol{B}=\mu_{o} \boldsymbol{j}$ can be also written as curl $\operatorname{curl} \boldsymbol{E}=-\frac{\partial \boldsymbol{j}}{\partial t}$, or in the matrix form $\hat{M} \boldsymbol{E}=i \omega \boldsymbol{j}$, where

$M^{j k}=\left|\begin{array}{ll}0 & 0 \\ 0 & k^{2}\end{array}\right|$,

and the current $j_{x}=\left[u_{p x}+n_{p} V_{p}+\alpha q\left(u_{h x}+n_{h} V_{h}\right)-(1+\alpha q)\right.$ $\left.\left(u_{e x}+n_{e} V_{e}\right)\right], j_{y}=\left[u_{p y}+\alpha q u_{h y}-(1+\alpha q) u_{e y}\right]$, or in the matrix form $\boldsymbol{j}_{i}=\hat{P}_{i} \boldsymbol{u}$, where

$$
\begin{aligned}
& P_{p}^{j k}=\left|\begin{array}{ll}
\frac{\omega}{\omega-k V_{p}} & 0 \\
0 & 1
\end{array}\right|, P_{h}^{j k}=\left|\begin{array}{ll}
\frac{\alpha q \omega}{\omega-k V_{p}} & 0 \\
0 & \alpha q
\end{array}\right|, \\
& P_{e}^{j k}=\left|\begin{array}{ll}
\frac{(1+\alpha q) \omega}{\omega-k V_{p}} & 0 \\
0 & 1+\alpha q
\end{array}\right| .
\end{aligned}
$$

The dispersion equation is then

$$
\operatorname{Det}\left[\hat{M}-i \omega\left(\hat{P}_{p} \hat{L}_{p}^{-1}+\hat{P}_{h} \hat{L}_{h}^{-1}-\hat{P}_{e} \hat{L}_{e}^{-1}\right]=0\right.
$$

Equation (37) reduces to a polynomial equation of fourth order for $\omega$. Using the current-free condition $\left(j_{x}=0\right)$ $(1+\alpha q) V_{e}=V_{p}+\alpha q V_{h}$, yields, in the long-wavelength limit $k \rightarrow 0$, the equation for the loci of stationary waves $\left(\frac{\omega}{k}=0\right)$

$V_{p}^{2}+\alpha \mu V_{h}^{2}=\beta_{p}+\alpha \mu \beta_{h}+(1+\alpha q) \beta_{e}+1$

or in dimensional form

$$
V_{p}^{2}+\alpha \mu V_{h}^{2}=c_{p}^{2}+\alpha \mu c_{h}^{2}+(1+\alpha q) c_{s}^{2}+V_{A p}^{2}
$$

In the case of an oblique magnetic field, all matrixes become of the third order, but the procedure for deriving the sonic locus remains similar.

Acknowledgements. E. M. Dubinin thanks the Deutsche Forschungsgemeinschaft and the Max-Planck-Gesellschaft for supporting this work by grant (WO 910/1-P).

Topical Editor I. A. Daglis thanks two referees for their help in evaluating this paper.

\section{References}

Baumgartel, K. and Sauer, K.: Interaction of a magnetized plasma stream with an immobile ion cloud, Ann. Geophys., 10, 763771, 1992,

http://www.ann-geophys.net/10/763/1992/.

Boesswetter, A., Bagdonat, T., Motschmann, U., and Sauer, K.: Plasma boundaries at Mars: a 3-D simulation study, Ann. Geophys., 22, 4363-4379, 2004, http://www.ann-geophys.net/22/4363/2004/.

Bertucci, C., Mazelle, C., Acuna, M., Russell, C. T., and Slavin, J. A.: Structure of the magnetic pileup boundary at Mars and Venus, J. Geophys. Res., 110, A01209, doi:10.1029/2004JA010592, 2005.

Coates, A. J.: Ionospheres and magnetospheres of comets, Adv. Space Res., 20(2), 255-266, 1997.

Dubinin, E., Sauer, K., McKenzie, J. F., and Chanteur, G.: Nonlinear waves and solitons propagating perpendicular to the magnetic field in bi-ion plasma with finite plasma pressure, Nonlin. Processes Geophys., 9, 87-99, 2002a.

Dubinin, E., Sauer, K., and McKenzie, J. F.: Solitons and oscillitons in cold bi-ion plasmas: A parameter study, J. Plasma Physics, 68, 27-52, 2002b.

Dubinin, E., Sauer, K., and McKenzie, J. F.: Solitons, oscillitons and stationary waves in a cold proton-alpha plasma, J. Geophys. Res., 107, 1295, doi:10.1029/2002JA009571, 2003a.

Dubinin, E., Sauer, K., and McKenzie, J. F.: Solitons, oscillitons and stationary waves in a warm proton-alpha plasma, J. Geophys. Res., 107, 1296, doi:10.1029/2002JA009572, 2003b.

McKenzie, J. F., Marsch, E., Baumgartel, K., and Sauer, K.: Wave and stability properties of multi-ion plasmas with applications to winds and flows, Ann. Geophys., 11, 341-353, 1993, http://www.ann-geophys.net/11/341/1993/.

McKenzie, J. F.: Stationary structures in multi-ion plasmas with differential streaming, J. Plasma Physics, 65, 181-195, 2001.

McKenzie, J. F., Sauer, K., and Dubinin, E.: Stationary waves in a bi-ion plasma transverse to the magnetic field, J. Plasma Physics, 65, 197-212, 2001.

McKenzie, J. F., Dubinin, E., Sauer, K., and Doyle, T. B.: Constants of the Motion for Nonlinear Stationary Waves in Complex Plasmas: A Unified Fluid Dynamic Viewpoint, J. Plasma Physics, 70, 431-462, 2004.

Rème, H., Sauvaud, J. A., d'Uston, C., et al.: General features of comet P/Halley: solar wind interaction from plasma measurements, Astron. Asrophys., 187, 33-38, 1987.

Rème, H., Mazelle, C., Sauvaud, J. A., d’Uston, C., et al.: Electron plasma environment at comet Grigg-Sjellerup: general observations and comparison with the environment of comet Halley, J. Geophys. Res., 98, 20 965-20 976, 1993. 
Sauer, K. and Dubinin, E.: The Martian "obstacle" boundary, Adv. Space Res., 26(10), 1633-1637, 2000.

Sauer, K., Roatsch, T., Baumgartel, K., and McKenzie, J. F.: Critical density layer as obstacle at solar wind-exospheric ion interaction, Geophys. Res. Lett., 19, 645-648, 1992.

Sauer, K., Bogdanov, A., Baumgartel, K., and Dubinin, E.: Plasma environment of comet Wirtanen during its low-activity stage, Planet. Space Sci., 44, 715-729, 1996.
Sauer, K., Dubinin, E., and McKenzie, J. F.: New type of soliton in bi-ion plasmas and possible implications, Geophys. Rev. Lett., 28, 3589-3592, 2001.

Sauer, K., Dubinin, E., and McKenzie, J. F.: Solitons and oscillitons in multi-ion plasmas, Nonlin. Processes Geophys., 10, 121-130, 2003 ,

http://www.nonlin-processes-geophys.net/10/121/2003/. 